\title{
Anisotropic symmetrization
}

\section{Symétrisation anisotrope}

\author{
Jean Van Schaftingen* \\ Département de Mathématique, Université catholique de Louvain, 2 chemin du Cyclotron, 1348 Louvain-la-Neuve, Belgium
}

Received 3 January 2005; received in revised form 31 May 2005; accepted 30 June 2005

Available online 6 December 2005

\begin{abstract}
The partial anisotropic symmetrization is defined, extending Steiner symmetrization and convex symmetrization. Inequalities of the type of Hardy-Littlewood, Pólya-Szegó and Klimov are proved for this symmetrization, while it is shown that Riesz-Sobolev rearrangement inequalities are not valid. Applications are given to the proof of isoperimetric inequalities, integral inequalities and existence and symmetry of solutions of variational problems.

(C) 2006 L'Association Publications de l'Institut Henri Poincaré. Published by Elsevier B.V. All rights reserved
\end{abstract}

\section{Résumé}

Nous définissons la symétrisation anisotrope partielle, qui est à la fois une extension de la symétrisation de Steiner et de la symétrisation convexe. Nous prouvons des inégalités du type Hardy-Littlewood, Pólya-Szegó et Klimov pour cette symétrisation, alors qu'elle ne vérifie pas d'inégalité de réarrangement du type Riesz-Sobolev. Nous donnons des applications à la preuve d'inégalités isopérimétriques et intégrales ainsi qu'à celle de l'existence et de la symétrie de solutions de problèmes anisotropes.

(C) 2006 L'Association Publications de l'Institut Henri Poincaré. Published by Elsevier B.V. All rights reserved

MSC: 26D50; 49Q20

Keywords: Symmetric rearrangement; Steiner symmetrization; Convex symmetrization; Pólya-Szegő inequality; Riesz-Sobolev rearrangement inequality; Isoperimetric inequality; Wulff's crystal; Hardy inequality; Sobolev inequality

\section{Introduction}

A symmetrization transforms functions into more symmetrical functions. This transformation preserves or decreases some integral functionals. This is useful to enquire about minimizers of a functional, which can then be sought among symmetrical functions. For example, consider the functional $I$, defined on $W_{0}^{1, p}(\Omega)$ by

$$
I(u)=\frac{\int_{\Omega}|\nabla u(x)|_{2}^{p} \mathrm{~d} x}{\left(\int_{\Omega}|u(x)|^{q} \mathrm{~d} x\right)^{p / q}} .
$$

\footnotetext{
* Tel.: +321047 31 80; fax: +3210472530.

E-mail address: vanschaftingen@math.ucl.ac.be (J. Van Schaftingen).
} 
If $\Omega=\mathrm{B}(0,1)$, then the Schwarz symmetrization $\cdot^{\star}$ maps any nonnegative function $u$ to a radial function $u^{\star}(x)=$ $v\left(|x|_{2}\right)$, where $v: \mathbb{R}^{+} \rightarrow \mathbb{R}^{+}$is a nonincreasing function, such that $I\left(u^{\star}\right) \leqslant I(u)[17,19,25,26]$. Therefore one can search for a minimizer among radial functions.

The symmetrization of functions remains possible whenever $\Omega$ has less symmetry. For example, if $\Omega=\mathrm{B}(0,1) \times$ $\mathbb{R}^{N-k} \subset \mathbb{R}^{N}$, then the Steiner symmetrization of $u$, also denoted by $\cdot^{\star}$ is a function $u^{\star}$ such that $I\left(u^{\star}\right) \leqslant I(u)$ and $u\left(x^{\prime}, x^{\prime \prime}\right)=v\left(|x|_{2}, x^{\prime \prime}\right)$ for some function $v: \mathbb{R}^{+} \times \mathbb{R}^{N-k} \rightarrow \mathbb{R}$ that does not increase with respect to its first argument.

The anisotropic symmetrization is a symmetrization adapted to anisotropic variational problems. In those problems, the function of the gradient in the functional does not depend on the Euclidian norm, but on another positively homogeneous function $H: \mathbb{R}^{N} \rightarrow \mathbb{R}^{+}$. Anisotropic problems have in general too small symmetry groups to obtain symmetry from uniqueness arguments as it is possible in the isotropic case. Therefore symmetrization seems to be the most natural way to prove symmetry of minimizers of anisotropic functionals.

Anisotropic problems arose at the beginning of the twentieth century in Wulff's work on crystal shapes and minimization of anisotropic surface tensions. He considered the minimization problem

$$
\min _{\mathcal{L}^{N}(\Omega)=1} \int_{\partial \Omega} H(v) \mathrm{d} \sigma,
$$

among sufficiently regular domains $\Omega$, and computed the solution whose optimality was proved by Dinghas and Taylor:

$$
\Omega=\left\{x \in \mathbb{R}^{N}: H^{\circ}(-x) \leqslant 1\right\}, \quad H^{\circ}(x)=\sup _{t \in \mathbb{R}^{N} \backslash\{0\}} \frac{\langle t, x\rangle}{H(t)} .
$$

This solution is unique up to translations [15]. This model explains the polyhedral shape of many crystals. The structure of the functional and of the solution are not the same, but are dual. A Schwarz anisotropic symmetrization was constructed for nonlinear variational problems by Alvino, Ferone, Lions and Trombetti [2] with the same duality relation. They proved Pólya-Szegố and Hardy-Littlewood inequalities for non-partial anisotropic symmetrizations.

In this paper, we study anisotropic symmetrization associated to a homogeneous convex function $G$ and its associated inequalities. Such hypotheses appear in other frameworks in the works of Taylor, Busemann, and Dacorogna and Pfister $[9,12,27]$. Our main objective is to define and understand partial anisotropic symmetrizations that generalize the convex symmetrization of Alvino, Ferone, Lions and Trombetti [2].

For any nonnegative measurable function $u: \mathbb{R}^{N} \rightarrow \overline{\mathbb{R}}^{+}$whose positive sublevel sets have finite measure and for any convex function $G \in \mathcal{H}\left(\mathbb{R}^{k}\right)$, a unique function $u^{*}: \mathbb{R}^{N} \rightarrow \overline{\mathbb{R}}^{+}$is defined such that $u^{*}\left(x^{\prime}, x^{\prime \prime}\right)=v\left(G\left(-x^{\prime}\right), x^{\prime \prime}\right)$, for any $x^{\prime} \in \mathbb{R}^{k}$ and $x^{\prime \prime} \in \mathbb{R}^{N-k}$, where the function $v: \mathbb{R}^{+} \times \mathbb{R}^{N-k} \rightarrow \overline{\mathbb{R}}^{+}$decreases with respect to its first argument and such that for any $c>0$ and $x^{\prime \prime} \in \mathbb{R}^{N-k}$,

$$
\mathcal{L}^{N-k}\left(\left\{x^{\prime}: u^{*}\left(x^{\prime}, x^{\prime \prime}\right)>c\right\}\right)=\mathcal{L}^{N-k}\left(\left\{x^{\prime}: u\left(x^{\prime}, x^{\prime \prime}\right)>c\right\}\right) .
$$

(See the beginning of Section 2 for precision on the notations.) This function $u^{*}$ is the anisotropic symmetrization of $u$ with respect to $G$. This transformation is a rearrangement in the sense of [6,29]. Therefore all classical integral inequalities follow easily, e.g. for any Borel measurable function $f: \mathbb{R} \times \mathbb{R}^{N-k} \rightarrow \mathbb{R}^{+}$such that $f(0, \cdot)=0$,

$$
\int_{\mathbb{R}^{N}} f\left(u^{*}, x^{\prime \prime}\right) \mathrm{d} x=\int_{\mathbb{R}^{N}} f\left(u, x^{\prime \prime}\right) \mathrm{d} x .
$$

Similarly,.$^{*}$ is a contraction in $L^{p}$ spaces (and many other spaces, see Proposition 2.29). The definitions and basic properties of the anisotropic symmetrizations are the object of Section 2.

Section 4 is devoted to convolution inequalities for the anisotropic symmetrization of the form

$$
\iint_{\mathbb{R}^{N}} u(x) v(y) w(x-y) \mathrm{d} x \mathrm{~d} y \leqslant \iint_{\mathbb{R}^{N}} \int_{\mathbb{R}^{N}} u^{*}(x) v^{*}(y) w^{*}(x-y) \mathrm{d} x \mathrm{~d} y .
$$

The conclusion is that such inequalities can occur only when the rearrangement is made with respect to an Euclidian gauge (Propositions 4.2 and 4.4). The same arguments show that the full Riesz-Sobolev rearrangement inequality does 
not hold for the spherical cap symmetrizations and the polarizations (Corollary 4.3). The proof of Proposition 4.4 uses dual characterizations of symmetrized functions studied in Section 3: For example if, for any $\varphi \in \mathcal{K}_{+}\left(\mathbb{R}^{N}\right)$,

$$
\int_{\mathbb{R}^{N}} \varphi u \mathrm{~d} x \leqslant \int_{\mathbb{R}^{N}} \varphi^{*} u \mathrm{~d} x
$$

then $u=u^{*}$ almost everywhere (Lemma 3.1). The case where $u$ is a measure is also investigated (Lemma 3.3).

Even if convolution inequalities do not hold for the anisotropic symmetrization, if $\varphi_{*}=-(-\varphi)^{*}$ and $\bar{\varphi}$ is the Fenchel transform of $\varphi$ (Definition 5.1), there are Klimov inequalities of the form

$$
\int_{\Omega} \overline{\varphi_{*}}\left(x^{\prime \prime}, u^{*}(x), \nabla u^{*}(x)\right) \mathrm{d} x \leqslant \int_{\Omega} \bar{\varphi}\left(x^{\prime \prime}, u(x), \nabla u(x)\right) \mathrm{d} x,
$$

(Theorem 6.9). In this inequality only the gauge $G$ appears, but the dual of Wulff's crystal is embedded inside the Fenchel transform.

The Pólya-Szegó inequality for anisotropic symmetrization can be stated as

$$
\int_{\Omega} J\left(x^{\prime \prime}, u^{*}, H\left(\nabla^{\prime} u^{*}\right), \nabla^{\prime \prime} u^{*}\right) \mathrm{d} x \leqslant \int_{\Omega} J\left(x^{\prime \prime}, u, H\left(\nabla^{\prime} u\right), \nabla^{\prime \prime} u\right) \mathrm{d} x,
$$

where $J$ is convex with respect to its last two variables and $G=H^{\circ}$ (Theorem 6.8). The left-hand side is not necessarily convex in $\nabla u$, since $H$ is not convex in general, but it is convex on the subset of gradients of symmetrized functions.

These results emphasize the local character of symmetries of crystals in contrast with the long-range of isotropic symmetry. Physically, this could be the fact that we observe anisotropic symmetries for crystals, whose energy is mainly an interface energy, but not for stars, whose energy depends of long-distance (gravitational) interaction terms.

The proof of these inequalities consists a generalization of an anisotropic inequality for Steiner symmetrization of Klimov [18] (Section 5), followed by a change of variable in order to return to anisotropic functionals (Section 6). The high degree of generality of the proof makes it appealing even for the isotropic symmetrization.

Applications of the previous inequalities are given as an anisotropic isoperimetric inequality (Theorem 7.2) and optimal constants for Sobolev and Hardy-Sobolev inequalities (Propositions 7.3 and 7.5). Finally, the existence and symmetry of solutions of two model anisotropic variational problems is showed (Propositions 7.6 and 7.9).

The definition of symmetrization is interesting also in the isotropic case because of its good pointwise behavior. As symmetrizations of sets were originally defined from compact sets to compact sets, they were nonexpansive mappings with respect to the Hausdorff distance. The symmetrization inequalities of Sarvas [23] for condensers or capacitors, defined by a compact set and an open set, required the extension of symmetrizations to open sets. Any extension to measurable sets, was only defined on almost every hyperplane. The use of Lebesgue's outer measure gives a precise definition of the symmetrized set which was needed in this paper since the Fenchel transform is sensitive to modifications on sets of measure zero.

This paper also clarifies the relationship between the different symmetrization inequalities by giving examples of symmetrizations for which Pólya-Szegó and Klimov inequalities hold but Riesz-Sobolev rearrangement inequalities do not hold.

\section{Definition and properties of symmetrizations}

In this section, the symmetrization with respect to a gauge is defined. Its basic properties, similar to those of the classical rearrangements, are studied.

Notation 2.1. For $f: X \rightarrow \overline{\mathbb{R}}$ and $c \in \overline{\mathbb{R}}$, let

$$
\{f<c\}=\{x \in X: f(x)<c\} \text {. }
$$

The characteristic function of a set $A$ is denoted $\chi_{A}$. The $N$-dimensional outer Lebesgue measure is denoted $\mathcal{L}^{N}$. The extended set of real numbers is $\overline{\mathbb{R}}=\mathbb{R} \cup\{+\infty,-\infty\}$. The set of compactly supported continuous functions is 
denoted $\mathcal{K}\left(\mathbb{R}^{N}\right)$, while $\mathcal{D}\left(\mathbb{R}^{N}\right)$ is the set of smooth functions with compact support. The subscript + denotes the subset of nonnegative functions of a function space. For $0 \leqslant k \leqslant N$ and $x=\left(x_{1}, \ldots, x_{N}\right) \in \mathbb{R}^{N}$, let $x^{\prime}=\left(x_{1}, \ldots, x_{k}\right)$ and $x^{\prime \prime}=\left(x_{k+1}, \ldots, x_{N}\right)$. Similarly, let $\nabla^{\prime} u=(\nabla u)^{\prime}$ and $\nabla^{\prime \prime} u=(\nabla u)^{\prime \prime}$.

Definition 2.2. Let $X$ be a vector space. The function $H: X \rightarrow \mathbb{R}$ belongs to $\mathcal{H}(X)$ if

(1) if $x \in X$ and $\lambda \geqslant 0$, then $H(\lambda x)=\lambda H(x)$,

(2) if $x \in X$ and $x \neq 0$, then $H(x)>0$,

(3) $H$ is lower semi-continuous.

Definition 2.3. The polar transform of $H \in \mathcal{H}\left(\mathbb{R}^{k}\right)$ is

$$
H^{\circ}: \mathbb{R}^{k} \rightarrow \mathbb{R}: H^{\circ}(t)=\sup _{x \in \mathbb{R}^{k}} \frac{\langle t, x\rangle}{H(x)},
$$

where $\langle t, x\rangle=\sum_{i=1}^{k} t_{i} x_{i}$.

Definition 2.4. The function $G: \mathbb{R}^{k} \rightarrow \mathbb{R}$ is a gauge if $G \in \mathcal{H}\left(\mathbb{R}^{k}\right)$ and $G$ is convex. For any gauge $G$, let

$$
K_{G}=\sqrt[k]{\frac{\mathcal{L}^{k}(\{G(-\cdot)<1\})}{\omega_{k}}},
$$

where $\omega_{k}=\mathcal{L}^{k}(\mathrm{~B}(0,1))$ is the volume of the unit ball in $\mathbb{R}^{k}$.

Remark 2.5. Any gauge $G$ is a continuous function, and $0<K_{G}<\infty$.

Example 2.6. If $H \in \mathcal{H}\left(\mathbb{R}^{k}\right)$, its polar transform $H^{\circ}$ is a gauge.

Definition 2.7. Let $G: \mathbb{R}^{k} \rightarrow \mathbb{R}^{+}$be a gauge. The anisotropic symmetrization (called convex symmetrization in [2]) of the set $A \subseteq \mathbb{R}^{k}$ with respect to $G$ is the set

$$
A^{*}=\left\{x \in \mathbb{R}^{k}: G(-x)<K_{G}^{-1}\left(\frac{\mathcal{L}^{k}(A)}{\omega_{k}}\right)^{1 / k}\right\} .
$$

Remark 2.8. The set $A^{*}$ is chosen among the sets $(\{G(-x)<r\})_{0 \leqslant r \leqslant \infty}$ so that $\mathcal{L}^{k}\left(A^{*}\right)=\mathcal{L}^{k}(A)$. The set $A$ does not have to be measurable.

Definition 2.9. Given a decomposition of $\mathbb{R}^{N}=L \times T$, and a gauge $G: L \rightarrow \mathbb{R}^{+}$, the $(G, L, T)$-anisotropic symmetrization of the set $A \subset \mathbb{R}^{N}$, is the unique set $A^{*}$ such that, for all $x^{\prime \prime} \in T$,

$$
\left[A^{*}\right]_{x^{\prime \prime}}=[A]_{x^{\prime \prime}} *,
$$

where $[B]_{x^{\prime \prime}}=\left\{x^{\prime} \in L:\left(x^{\prime}, x^{\prime \prime}\right) \in B\right\}$ and the symmetrization on the right-hand side comes from Definition 2.7.

The symmetrization of a finite-measure set $A \subset \mathbb{R}^{N}$ with respect to a gauge $G: \mathbb{R}^{k} \rightarrow \mathbb{R}^{+}$is the $\left(G, \mathbb{R}^{k} \times\{0\},\{0\} \times\right.$ $\mathbb{R}^{N-k}$ )-anisotropic symmetrization of $A$.

Remark 2.10. Even when $A$ is measurable, $[A]_{x^{\prime \prime}}$ is not measurable in general. Therefore Definition 2.7 embraces nonmeasurable sets.

Remark 2.11. The result $A^{*}$ of the anisotropic symmetrization with respect to the gauge $G$ has a cylindrical geometry:

$$
A^{*}=\left\{\left(x^{\prime}, x^{\prime \prime}\right) \in \mathbb{R}^{N}: G\left(-x^{\prime}\right)<K_{G}^{-1}\left(\frac{\mathcal{L}^{k}\left([A]_{x^{\prime \prime}}\right)}{\omega_{k}}\right)^{1 / k}\right\} .
$$


Example 2.12. The $(k, N)$-Steiner symmetrization with respect to the subspace $T \subseteq \mathbb{R}^{N}$, is the $\left(G, T^{\perp}, T\right)$-anisotropic symmetrization, where $G: x^{\prime \prime} \mapsto\left|x^{\prime \prime}\right|_{2}$. The Steiner symmetrization with respect to $\mathbb{R}^{N}$ is the Schwarz symmetrization (see e.g. [4,6,23]).

Example 2.13. If $G: \mathbb{R}^{N} \rightarrow \mathbb{R}^{+}$is a gauge and is an even function, then the anisotropic symmetrization with respect to $G$ is the convex symmetrization with respect to $G$ of Alvino, Ferone, Lions and Trombetti [2].

The following proposition summarizes the properties of anisotropic symmetrization of sets.

Proposition 2.14. Let $*^{*}$ be a $(G, L, T)$-anisotropic symmetrization on $\mathbb{R}^{N}$.

(1) (Monotonicity) If $A \subseteq B \subseteq \mathbb{R}^{N}$, then $A^{*} \subseteq B^{*}$.

(2) (Interior continuity) If $\left(A_{n}\right)_{n \in \mathbb{N}}$ is an increasing sequence of subsets of $\mathbb{R}^{N}$ (i.e. $A_{n} \subseteq A_{n+1}$ ), then

$$
\left(\bigcup_{n \in \mathbb{N}} A_{n}\right)^{*}=\bigcup_{n \in \mathbb{N}} A_{n}^{*}
$$

(3) (Preservation of measure) If $A \subset \mathbb{R}^{N}$ is measurable, then $A^{*}$ is measurable and $\mathcal{L}^{N}(A)=\mathcal{L}^{N}\left(A^{*}\right)$.

(4) If $A \subset \mathbb{R}^{N}$ is open, then $A^{*}$ is open.

Remark 2.15. Whereas the continuity of symmetrization held in previous works only up to sets of zero measure $[6,23]$, this definition ensures interior continuity. The exterior continuity (Property (2) with reversed inclusions) still holds up to sets of zero measure, but it is not used in the sequel. This property was true already for the definitions of the Schwarz symmetrization which mapped all sets to open sets (see e.g. [19]).

Remark 2.16. Part (3) remains true when $A$ is not measurable if $T=\{0\}$, by definition of the anisotropic symmetrization. If $T \neq\{0\}$, then part (3) implies $\mathcal{L}^{N}\left(A^{*}\right) \leqslant \mathcal{L}^{N}(A)$, and for some nonmeasurable sets $A \subset \mathbb{R}^{N}$, the inequality is strict.

Proof. The notations of Definition 2.9 are used throughout the proof. Part (1) comes from the monotonicity of outer measures: for any $x^{\prime \prime} \in T$, since $[A]_{x^{\prime \prime}} \subset[B]_{x^{\prime \prime}}, \mathcal{L}^{k}\left([A]_{x^{\prime \prime}}\right) \leqslant \mathcal{L}^{k}\left([B]_{x^{\prime \prime}}\right)$, and $\left[A^{*}\right]_{x^{\prime \prime}} \subset\left[B^{*}\right]_{x^{\prime \prime}}$. It is then clear that $A^{*} \subset B^{*}$.

If $\left(A_{n}\right)$ is any sequence satisfying the hypotheses of (2), for any $x^{\prime \prime} \in T$, by an elementary property of Lebesgue's outer measure,

$$
\lim _{n \rightarrow \infty} \mathcal{L}^{k}\left(\left[A_{n}\right]_{x^{\prime \prime}}\right)=\mathcal{L}^{k}\left(\bigcup_{n \in \mathbb{N}}\left[A_{n}\right]_{x^{\prime \prime}}\right)=\mathcal{L}^{k}\left([A]_{x^{\prime \prime}}\right) .
$$

(This is a consequence of monotonicity and countable subadditivity of outer measures, see e.g. [14, 2.1.5] for a proof.) Since the sequence $\left(\left[A_{n}\right]_{x^{\prime \prime}}\right)_{n \in \mathbb{N}}$ is increasing, by Definition 2.7, $\bigcup_{n \in \mathbb{N}}\left(\left[A_{n}\right]_{x^{\prime \prime}}{ }^{*}\right)=[A]_{x^{\prime \prime}}{ }^{*}$. By Definition 2.9, $\bigcup_{n \in \mathbb{N}} A_{n}{ }^{*}=A^{*}$.

Part (3) is a consequence of Remark 2.11 and of Fubini's theorem (see e.g. [14, 2.6.2]):

$$
\mathcal{L}^{N}\left(A^{*}\right)=\int_{T} \mathcal{L}^{k}\left([A]_{x^{\prime \prime}}{ }^{*}\right) \mathrm{d} x^{\prime \prime}=\int_{T} \mathcal{L}^{k}\left([A]_{x^{\prime \prime}}\right) \mathrm{d} x^{\prime \prime}=\mathcal{L}^{N}(A) .
$$

Part (4) relies on Remark 2.11. If the set $A$ is open, the right-hand side of the inequality inside (2.1) is lower semi-continuous, whence the symmetrized set $A^{*}$ is open.

Following $[6,29,30]$, the symmetrization is extended from sets to functions.

Definition 2.17. The $(G, L, T)$-anisotropic (decreasing) symmetrization $*^{*}$ of a function $u: \mathbb{R}^{N} \rightarrow \overline{\mathbb{R}}$ is

$$
u^{*}: \mathbb{R}^{N} \rightarrow \overline{\mathbb{R}}: x \mapsto u^{*}(x)=\sup \left\{c \in \overline{\mathbb{R}}: x \in\{u>c\}^{*}\right\} .
$$


Remark 2.18. Since $\chi\left(A^{*}\right)=\left(\chi_{A}\right)^{*}$, the symmetrization of functions is an extension of the symmetrization of sets.

Notation 2.19. For a function $u$ and a sequence of functions $\left(u_{n}\right)_{n \in \mathbb{N}}$ from a set $X$ to $\overline{\mathbb{R}}$, we write $u_{n} \nearrow u$ if for all $x \in X, \lim _{n \rightarrow \infty} u_{n}(x)=u(x)$ and for all $n \in \mathbb{N}, u_{n}(x) \leqslant u_{n+1}(x)$. Similarly, $\varphi_{n} \searrow \varphi$ if $-\varphi_{n} \nearrow-\varphi$.

The simplest properties of symmetrization of functions are consequence of the corresponding properties of symmetrization of sets $[6,29]$.

Proposition 2.20. Let $*^{*}$ be a $(G, L, T)$-anisotropic symmetrization.

(1) For any $u: \mathbb{R}^{N} \rightarrow \overline{\mathbb{R}}$

$$
u^{*}(x)=\sup \left\{c \in \mathbb{R}: x \in\{u \geqslant c\}^{*}\right\} .
$$

(2) For any $c \in \overline{\mathbb{R}}$ and $u: \mathbb{R}^{N} \rightarrow \overline{\mathbb{R}}$,

$$
\{u>c\}^{*}=\left\{u^{*}>c\right\} .
$$

(3) Let $u, v: \mathbb{R}^{N} \rightarrow \overline{\mathbb{R}}$. If $u \leqslant v$, then $u^{*} \leqslant v^{*}$.

(4) If $\left(u_{n}\right)_{n \in \mathbb{N}}$ is a sequence of functions from $\mathbb{R}^{N}$ to $\overline{\mathbb{R}}$, and $u_{n} \nearrow u$, then

$$
u_{n}^{*} \nearrow u^{*} \text {. }
$$

Remark 2.21. Part (1) is Hildén's definition of the Schwarz symmetrization of a function.

Remark 2.22. Part (2) means that if .* is the $(G, L, T)$-symmetrization, the hypograph of the symmetrization is the symmetrization of the hypograph:

$$
\left\{(x, c) \in \mathbb{R}^{N} \times \mathbb{R}: u^{*}(x)>c\right\}=\left\{(x, c) \in \mathbb{R}^{N} \times \mathbb{R}: u(x)>c\right\}^{*} .
$$

(The symmetrization on the right-hand side is the $(G, L \times\{0\}, T \times \mathbb{R})$-anisotropic symmetrization in $\mathbb{R}^{N} \times \mathbb{R}$.) This is essentially Pólya and Szegô's definition of the symmetrization of a function [22].

Remark 2.23. Part (2) implies in particular that if $u(x)>c$ for almost every $x \in \mathbb{R}^{N}$, then $u^{*}(x)>c$ for all $x \in \mathbb{R}^{N}$. If the function $u$ does not take the value $-\infty$, neither does its symmetrization $u^{*}$.

Remark 2.24. The equality of sets in part (2) holds pointwise. This is not the case for most of the usual definitions of rearrangements, for which the equality in part (2) holds only up to a set of zero measure. This comes from the fact our definition of symmetrization ensures interior continuity in a pointwise sense.

Part (2) holds with the strict inequality sign, but not with the non-strict inequality (if $u^{*}$ is a nonconstant continuous function, then for some $c$ the set $\left\{u^{*} \geqslant c\right\}$ is closed, while $\{u \geqslant c\}^{*}$ is not closed).

Remark 2.25. In part (4), $\lim _{n \rightarrow \infty} u_{n}(x)=u(x)$ holds everywhere. That is crucial in Section 5, since the Fenchel transform is continuous for increasing sequences converging everywhere.

Proof of Proposition 2.20. Part (3) is a consequence of the monotonicity of the anisotropic symmetrization.

For part (1), let $\tilde{u}(y)$ denote the right-hand side of the inequality. It is clear from monotonicity of the symmetrization that for any $\varepsilon>0, u^{*}(y) \leqslant \tilde{u}(y) \leqslant(u+\varepsilon)^{*}(y)=u^{*}(y)+\varepsilon$. The conclusion follows as $\varepsilon \rightarrow 0$.

By the interior continuity of the symmetrization of sets (Proposition 2.14), for any $c \in \mathbb{R}$,

$$
\{u>c\}^{*} \subseteq\left\{u^{*}>c\right\} \subseteq \bigcup_{n \in \mathbb{N}}\left\{u>c+\frac{1}{n}\right\}^{*}=\left(\bigcup_{n \in \mathbb{N}}\left\{u>c+\frac{1}{n}\right\}\right)^{*}=\{u>c\}^{*} .
$$

The proof is the same for $c=-\infty$, provided $c+1 / n$ is replaced by $-n$, while for $c=+\infty$, both sides are the empty set. This proves part (2).

Part (4) is a consequence of the interior continuity of symmetrization of sets (Proposition 2.14, part (2)) and of the description of the symmetrization of a function of part (2). 
Remark 2.26. The proof of Proposition 2.20 only relies on the corresponding properties for the symmetrization of sets (Proposition 2.14, parts (1) and (2)).

The preservation of measure for the symmetrization of sets has as counterpart integral equalities and inequalities for the symmetrization of functions. A natural class for studying integrals is the class of functions vanishing at the infinity (see Lieb and Loss [19]). This class contains the functions spaces $L^{p}\left(\mathbb{R}^{N}\right)$ and $C_{0}\left(\mathbb{R}^{N}\right)$ (continuous functions such that $\left.\lim _{|x|_{2} \rightarrow \infty} u(x)=0\right)$.

Definition 2.27. A measurable function $u: \mathbb{R}^{N} \rightarrow \overline{\mathbb{R}}$ vanishes at the infinity with respect to a $k$-dimensional linear subspace $L \subset \mathbb{R}^{N}$ if for all $c>0$ and $x \in \mathbb{R}^{N}, \mathcal{L}^{k}(\{u>c\} \cap(L+x))<\infty$. We also say that $u$ vanishes at the infinity with respect to the $(G, L, T)$-anisotropic symmetrization.

Proposition 2.28 ((Cavalieri principle)). Let $\cdot^{*}$ be the anisotropic symmetrization with respect to a gauge $G: \mathbb{R}^{k} \rightarrow$ $\mathbb{R}^{+}$.

If $u: \mathbb{R}^{N} \rightarrow \overline{\mathbb{R}}^{+}$vanishes at the infinity with respect to ${ }^{*}$ and $f: \mathbb{R} \times \mathbb{R}^{N-k} \rightarrow \overline{\mathbb{R}}^{+}$is a Borel measurable function such that $f\left(0, x^{\prime \prime}\right)=0$ for almost every $x^{\prime \prime} \in \mathbb{R}^{N-k}$, then

$$
\int_{\mathbb{R}^{N}} f\left(u^{*}, x^{\prime \prime}\right) \mathrm{d} x=\int_{\mathbb{R}^{N}} f\left(u, x^{\prime \prime}\right) \mathrm{d} x .
$$

(Hardy-Littlewood inequality) Let $F: \mathbb{R} \times \mathbb{R} \times \mathbb{R}^{N-k} \rightarrow \mathbb{R}$ be a function such that

(i) $F(s, t, \cdot)$ is measurable for every $(s, t) \in \mathbb{R} \times \mathbb{R}$,

(ii) $F\left(\cdot, \cdot, x^{\prime \prime}\right)$ is continuous for almost every $x^{\prime \prime} \in T$,

(iii) for almost every $x^{\prime \prime} \in \mathbb{R}^{N-k}$, and for any $a, b, c, d \in \mathbb{R}$, if $a \leqslant b, c \leqslant d$,

$$
F\left(a, c, x^{\prime \prime}\right)+F\left(b, d, x^{\prime \prime}\right) \geqslant F\left(a, d, x^{\prime \prime}\right)+F\left(b, c, x^{\prime \prime}\right) .
$$

If $u, v$ are nonnegative measurable functions defined on $\mathbb{R}^{N}$ and $F\left(u, 0, x^{\prime \prime}\right), F\left(0, v, x^{\prime \prime}\right)$ and $F\left(u, v, x^{\prime \prime}\right)$ are summable, then

$$
\int_{\mathbb{R}^{N}} F\left(u, v, x^{\prime \prime}\right) \mathrm{d} x \leqslant \int_{\mathbb{R}^{N}} F\left(u^{*}, v^{*}, x^{\prime \prime}\right) \mathrm{d} x .
$$

Proof. The result is true without any dependence on $x^{\prime \prime}$ (see [21,29]). For any $x^{\prime \prime}$, there holds

$$
\int_{\mathbb{R}^{N-k}} f\left(u^{*}\left(x^{\prime}, x^{\prime \prime}\right), x^{\prime \prime}\right) \mathrm{d} x^{\prime}=\int_{\mathbb{R}^{N-k}} f\left(u\left(x^{\prime}, x^{\prime \prime}\right), x^{\prime \prime}\right) \mathrm{d} x^{\prime} .
$$

Since $f$ is a Borel measurable function, $f(u(\cdot), \cdot)$ is measurable and is almost everywhere equal to a Borel measurable function. The result comes from the application of Fubini's theorem.

For the second inequality uses the fact that $F$ is a Carathédory function and that such functions are almost everywhere equal to a Borel measurable function. The conclusion comes from the corresponding inequality in $[10,30]$ and Fubini's theorem.

Proposition 2.29. Let $\cdot^{*}$ be the anisotropic symmetrization with respect to $G$ and $g: \mathbb{R} \times \mathbb{R}^{N-k} \rightarrow \overline{\mathbb{R}}^{+}$. Suppose that for almost every $x^{\prime \prime} \in \mathbb{R}^{N-k}, g\left(\cdot, x^{\prime \prime}\right)$ is a convex and lower semi-continuous function and $g\left(0, x^{\prime \prime}\right)=0$, and that for all $s \in \mathbb{R}, g\left(s, x^{\prime \prime}\right)$ is measurable. If $u$ and $v$ are measurable functions, then

$$
\int_{\mathbb{R}^{N}} g\left(u^{*}(x)-v^{*}(x), x^{\prime \prime}\right) \mathrm{d} x \leqslant \int_{\mathbb{R}^{N}} g\left(u(x)-v(x), x^{\prime \prime}\right) \mathrm{d} x .
$$

For any $1 \leqslant p \leqslant+\infty$ and for any measurable functions $u$ and $v$

$$
\left\|u^{*}-v^{*}\right\|_{p} \leqslant\|u-v\|_{p} .
$$


Remark 2.30. There are no integrability assumptions in this proposition.

Proof. By Fubini's theorem, we have to prove

$$
\int_{\mathbb{R}^{k}} \int_{\mathbb{R}^{N-k}} g\left(u^{*}-v^{*}, x^{\prime \prime}\right) \mathrm{d} x \leqslant \int_{\mathbb{R}^{k}} \int_{\mathbb{R}^{N-k}} g\left(u-v, x^{\prime \prime}\right) \mathrm{d} x .
$$

The inequality will be proved for the interior integral. Without loss of generality we can thus assume $k=N$ and $g\left(s, x^{\prime \prime}\right)=g(s)$. Let

$$
c=\inf \left\{s: \mathcal{L}^{N}(\{u>s\})<\infty\right\}, \quad d=\inf \left\{s: \mathcal{L}^{N}(\{v>s\})<\infty\right\} .
$$

If $g(c-d)>0$, then, without loss of generality, we can assume that $c>d$. By definition of $c$ and $d$, for any $\varepsilon>0$, $\mathcal{L}^{N}(\{v>d+\varepsilon\})<+\infty$ and $\mathcal{L}^{N}(\{u>c-\varepsilon\})=+\infty$, hence the measure of the set $\Omega_{\varepsilon}=\{u>c-\varepsilon\} \cap\{v \leqslant d+\varepsilon\}$ is infinite. Furthermore, for any $x \in \Omega_{\varepsilon}, u(x)-v(x)>c-d-2 \varepsilon$. Since $g$ is convex and $g(0)=0$, it is increasing on $\mathbb{R}^{+}$. Hence, for $\varepsilon \leqslant \frac{c-d}{2}$,

$$
g(u(x)-v(x)) \geqslant g(c-d-2 \varepsilon) .
$$

Since $g$ is lower semi-continuous, the right-hand side of (2.2) is positive for sufficiently small $\varepsilon$. This means

$$
\int_{\mathbb{R}^{N}} g(u(x)-v(x)) \mathrm{d} x=+\infty .
$$

The inequality is then trivial.

If $g(c-d)=0$, without loss of generality we can assume that $c=d=0$. If $g$ is continuous, the function $F:(s, t) \mapsto$ $-g(s-t)$ verifies the hypotheses of the last part of Proposition 2.28. If $u_{n}=\min (n, \max (u-1 / n, 0))$, and $v_{n}=$ $\min (n, \max (v-1 / n, 0))$, then $g\left(u_{n}\right), g\left(-v_{n}\right)$ and $g\left(u_{n}-v_{n}\right)$ are summable and, by Proposition 2.28,

$$
\int_{\mathbb{R}^{N}} g\left(u_{n}^{*}(x)-v_{n}^{*}(x)\right) \mathrm{d} x \leqslant \int_{\mathbb{R}^{N}} g\left(u_{n}(x)-v_{n}(x)\right) \mathrm{d} x .
$$

Furthermore, $u_{n} \nearrow u^{+}, v_{n} \nearrow v^{+}$and $g\left(u_{n}-v_{n}\right) \nearrow g\left(u^{+}-v^{+}\right) \leqslant g(u-v)$, whence $u_{n}^{*} \nearrow u^{*}$, and $v_{n}^{*} \nearrow v^{*}$. Moreover, $\liminf _{n \rightarrow \infty} g\left(u_{n}^{*}(x)-v_{n}^{*}(x)\right) \geqslant g\left(u^{*}(x)-v^{*}(x)\right)$ since $g$ is continuous. Fatou's lemma and Levi's monotone convergence theorem bring the conclusion. If $g$ is not continuous, it can be approximated by an increasing sequence of continuous convex functions and Levi's monotone convergence theorem gives the conclusion.

The second part is a consequence of the first part, with $g=|t|^{p}$ if $1 \leqslant p<+\infty$. If $p=+\infty$, let $g(t)=0$ for $t \leqslant\|u-v\|_{\infty}$ and $g(t)=+\infty$ else.

The anisotropic symmetrizations can be defined for functions which are defined on totally invariant sets.

Definition 2.31. A set $\Omega \subset \mathbb{R}^{N}$ is totally invariant with respect to a hyperplane $L$ if $\Omega+L=\Omega$. The set $\Omega$ is totally invariant with respect to the $(G, L, T)$-anisotropic symmetrization if it is totally invariant with respect to $L$.

A function $f: \mathbb{R}^{N} \rightarrow \overline{\mathbb{R}}$ is totally invariant with respect to a hyperplane $L$ if $f(x+l)=f(x)$ for any $l \in L$.

If $\Omega$ is totally invariant with respect to the $(G, L, T)$-anisotropic symmetrization $\cdot^{*}$, then for any $A \subset \mathbb{R}^{N}$, $(A \cap \Omega)^{*}=A^{*} \cap \Omega$.

Definition 2.32. If $*^{*}$ is an anisotropic symmetrization, $\Omega$ is totally invariant with respect to ${ }^{*}$, then the symmetrization of $u: \Omega \rightarrow \overline{\mathbb{R}}$ is $u^{*}=\left.\tilde{u}^{*}\right|_{\Omega}$, where $\tilde{u}$ denotes an extension of $u$ to $\mathbb{R}^{N}$.

The definition of $u^{*}$ does not depend on the extension $\tilde{u}$. All the previous results remain valid with $\Omega$ in place of $\mathbb{R}^{N}$, provided the set $\Omega$ is measurable for the integral inequalities.

The increasing symmetrization is a natural counterpart to the decreasing symmetrization. 
Definition 2.33. The $(G, L, T)$-anisotropic increasing symmetrization of a function $\varphi: \mathbb{R}^{N} \rightarrow \overline{\mathbb{R}}$ is

$$
\varphi_{*}(y)=\inf \left\{s \in \mathbb{R}: y \in\{\varphi<s\}^{*}\right\} .
$$

The increasing and decreasing anisotropic symmetrization are essentially the same transformation.

Proposition 2.34. For any function $\varphi: \mathbb{R}^{N} \rightarrow \overline{\mathbb{R}}$

$$
\varphi_{*}(y)=-(-\varphi)^{*}(y) .
$$

Remark 2.35. This means that all the properties of the decreasing symmetrization are true for the increasing symmetrization up to obvious modifications.

Proof. For any $y \in \mathbb{R}^{N}$,

$$
\begin{aligned}
\varphi_{*}(y) & =\inf \left\{s \in \mathbb{R}: y \in\{\varphi<s\}^{*}\right\}=-\sup \left\{c \in \mathbb{R}: y \in\{\varphi<-c\}^{*}\right\} \\
& =-\sup \left\{c \in \mathbb{R}: y \in\{-\varphi>c\}^{*}\right\}=-(-\varphi)^{*}(y) .
\end{aligned}
$$

\section{Dual characterization of symmetrized functions}

This section is devoted to dual characterizations of symmetrized functions that are used in the proof of Proposition 4.4 .

Lemma 3.1. Let ${ }^{*}$ be an anisotropic symmetrization and let $u \in L_{+}^{1}\left(\mathbb{R}^{N}\right)$. If for any compact set $K \subset \mathbb{R}^{N}$,

$$
\int_{K} u \mathrm{~d} x \leqslant \int_{K^{*}} u \mathrm{~d} x,
$$

then $u=u^{*}$ almost everywhere.

If, for any $\varphi \in \mathcal{K}_{+}\left(\mathbb{R}^{N}\right)$,

$$
\int_{\mathbb{R}^{N}} \varphi u \mathrm{~d} x \leqslant \int_{\mathbb{R}^{N}} \varphi^{*} u \mathrm{~d} x,
$$

then $u=u^{*}$ almost everywhere.

Remark 3.2. This lemma is reminiscent of the bathtub principle and of the necessary condition of equality of the Hardy-Littlewood inequality (see [19]).

Proof. If the inequality (3.1) holds for any compact set $K$, then it holds also for any measurable set $B$ by interior continuity of the anisotropic symmetrization and by Levi's monotone convergence theorem. The inequality (3.1) is equivalent to

$$
\int_{B \backslash B^{*}} u \mathrm{~d} x \leqslant \int_{B^{*} \backslash B} u \mathrm{~d} x,
$$

for any measurable set $B$. For $c \in \mathbb{R}$, let $B=\{u>c\}$. Then the inequality (3.3) holds if and only if $\mathcal{L}^{N}\left(B \backslash B^{*}\right)=0$. The function $u$ is then almost everywhere equal to a function $\tilde{u}$ such that $\{\tilde{u}>c\}=\{u>c\}^{*}=\left\{u^{*}>c\right\}$. By the characterization of the symmetrization by sublevel sets (Proposition 2.20), $\tilde{u}=u^{*}$.

Suppose now that the inequality (3.2) holds for any $\varphi \in \mathcal{K}_{+}\left(\mathbb{R}^{N}\right)$. Let $K \subset \mathbb{R}^{N}$ be compact. Let $\varphi_{n}=\chi_{\mathrm{B}(0,1 / n)} * \chi_{K}$, where $*$ denotes the convolution product. Then $\varphi_{n}$ is continuous, $\varphi_{n} \rightarrow \chi_{K}$ in $L^{1}\left(\mathbb{R}^{N}\right)$. Hence, by Proposition 2.29 , $\varphi_{n}^{*} \rightarrow \chi_{K^{*}}$ in $L^{1}\left(\mathbb{R}^{N}\right)$. Up to a subsequence $\varphi_{n}^{*}(x) \rightarrow \chi_{B^{*}}(x)$ and $\varphi_{n}^{*}(x) \leqslant 1$ for almost every $x \in \mathbb{R}^{N}$. Hence by Lebesgue's dominated convergence theorem, the inequality (3.1) holds, and the conclusion comes from the first part of the proof. 
Lemma 3.3. Let $G: \mathbb{R}^{k} \rightarrow \mathbb{R}^{+}$be a gauge, .* be the anisotropic symmetrization with respect to $G$. Suppose $\mu$ is a nonnegative Radon measure such that for any $\varphi \in \mathcal{K}_{+}\left(\mathbb{R}^{k}\right)$,

$$
\int_{\mathbb{R}^{k}} \varphi \mathrm{d} \mu \leqslant \int_{\mathbb{R}^{k}} \varphi^{*} \mathrm{~d} \mu
$$

Then there exists $w \in L_{+}^{1}\left(\mathbb{R}^{k}\right)$ and $a \geqslant 0$ such that $w=w^{*}$ and

$$
\int_{\mathbb{R}^{k}} \varphi \mathrm{d} \mu=a \varphi(0)+\int_{\mathbb{R}^{k}} w \varphi \mathrm{d} x
$$

Proof. Let $\psi \in \mathcal{D}_{+}(\mathrm{B}(0,1))$ and $\psi_{\rho}(x)=\psi^{*}\left(\rho^{-1} x\right)$. Fix $x \in \mathbb{R}^{k} \backslash\{0\}$. For any $\rho>0$ and $\varepsilon>0$, if $\rho+\varepsilon<|x|_{2}$, then

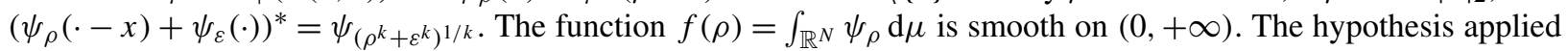
to the function $\psi_{\rho}(\cdot-x)+\psi_{\varepsilon}(\cdot)$ gives

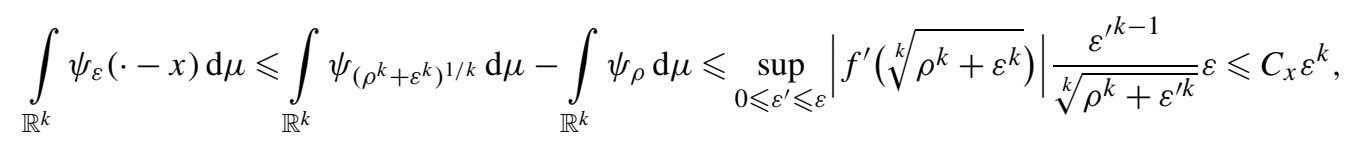

where $C_{x}$ is a constant that depends only on $x$ and $\rho$ for $\varepsilon$ in a neighborhood of 0 . Hence $\mu$ is absolutely continuous on $\mathbb{R}^{k} \backslash\{0\}$ and by the Radon-Nikodym Theorem of decomposition of a measure, the support of the singular part of $\mu$ lies in the set $\{0\}$. Therefore, there exists $a \in \mathbb{R}$ and $w \in L^{1}\left(\mathbb{R}^{k}\right)$ such that

$$
\int_{\mathbb{R}^{k}} \psi \mathrm{d} \mu=a \psi(0)+\int_{\mathbb{R}^{k}} w \mathrm{~d} x
$$

Since $w \in L^{1}\left(\mathbb{R}^{k}\right)$, for any fixed $x \neq 0$,

$$
0=\lim _{\rho \rightarrow 0} \int_{\mathbb{R}^{k}} \psi_{\rho}(\cdot-x) \mathrm{d} \mu \leqslant \lim _{\rho \rightarrow 0} \int_{\mathbb{R}^{k}} \psi_{\rho} \mathrm{d} \mu=a \psi(0) .
$$

Because $\psi(0)>0, a \geqslant 0$.

Now, let $\varphi \in \mathcal{K}\left(\mathbb{R}^{k} \backslash\{0\}\right)$. For sufficiently small $\varepsilon>0$, it is clear that

$$
\left(\varphi+\varphi^{*}\left(\frac{\cdot}{\varepsilon}\right)\right)^{*}=\varphi^{*}\left(\frac{\cdot}{\left(1+\varepsilon^{k}\right)^{1 / k}}\right) .
$$

Hence,

$$
\begin{aligned}
\int_{\mathbb{R}^{k}}\left(\varphi(x)+\varphi^{*}\left(\frac{x}{\varepsilon}\right)\right) w(x) \mathrm{d} x+a \varphi^{*}(0) & =\int_{\mathbb{R}^{k}} \varphi(x)+\varphi^{*}\left(\frac{x}{\varepsilon}\right) \mathrm{d} \mu \leqslant \int_{\mathbb{R}^{k}} \varphi^{*}\left(\frac{x}{\left(1+\varepsilon^{k}\right)^{1 / k}}\right) \mathrm{d} \mu \\
& =\int_{\mathbb{R}^{k}} \varphi^{*}\left(\frac{x}{\left(1+\varepsilon^{k}\right)^{1 / k}}\right) w(x) \mathrm{d} x+a \varphi^{*}(0) .
\end{aligned}
$$

If $\varepsilon \rightarrow 0$, inequality (3.2) follows. For a general $\varphi \in \mathcal{K}_{+}\left(\mathbb{R}^{k}\right)$, there exists a sequence $\left(\varphi_{m}\right)_{m \in \mathbb{N}}$ in $\mathcal{K}_{+}\left(\mathbb{R}^{k} \backslash\{0\}\right)$ such that $\varphi_{m} \nearrow \varphi$ almost everywhere. As $m \rightarrow \infty$, the inequality (3.2) follows. The conclusion comes from Lemma 3.1.

\section{Riesz-Sobolev rearrangement inequalities}

In this section we prove that the Riesz-Sobolev rearrangement inequalities do not hold for an anisotropic symmetrizations unless it is the classical Steiner symmetrization. That is the crucial difference between Steiner and anisotropic symmetrizations. This justifies the approach of the following sections for the Pólya-Szegó inequalities. 
If $\cdot{ }^{\star}$ denotes a Steiner symmetrization, the Riesz-Sobolev inequality

$$
\int_{\mathbb{R}^{N}} \int_{\mathbb{R}^{N}} u(x) v(y) w(x-y) \mathrm{d} x \mathrm{~d} y \leqslant \iint_{\mathbb{R}^{N}} \int_{\mathbb{R}^{N}} u^{\star}(x) v^{\star}(y) w^{\star}(x-y) \mathrm{d} x \mathrm{~d} y
$$

holds for any nonnegative functions vanishing at infinity $u, v$ and $w$ (see Brascamp, Lieb and Luttinger [5], and Lieb and Loss [19]).

Lemma 4.1. Let $A \subset \mathbb{R}^{N}$ and $E \subset \mathbb{R}^{N}$. If $E$ is an ellipsoid, $A$ is measurable, $\mathcal{L}^{N}(A)=\mathcal{L}^{N}(E)$, and

$$
\int_{\mathbb{R}^{2 N}} \chi_{E}(x) \chi_{E}(y) \chi_{E}(x-y) \mathrm{d} x \mathrm{~d} y \leqslant \int_{\mathbb{R}^{2 N}} \chi_{A}(x) \chi_{A}(y) \chi_{A}(x-y) \mathrm{d} x \mathrm{~d} y,
$$

then $A$ is an ellipsoid centered around the origin up to a set of measure 0.

Proof. Since the inequality remains invariant through affine change of variables, suppose $E=\mathrm{B}(0,1)$ without loss of generality. Let $\cdot^{\star}$ be the Schwarz symmetrization. Then $A^{\star}=\mathrm{B}(0,1)=E^{\star}=E$. Since the inequality (4.1) holds for the Schwarz symmetrization,

$$
\begin{aligned}
\int_{\mathbb{R}^{N}} \int_{\mathbb{R}^{N}} \chi_{E}(x) \chi_{E}(y) \chi_{E}(x-y) \mathrm{d} x \mathrm{~d} y & \leqslant \int_{\mathbb{R}^{N}} \int_{\mathbb{R}^{N}} \chi_{A}(x) \chi_{A}(y) \chi_{A}(x-y) \mathrm{d} x \mathrm{~d} y \\
& \leqslant \iint_{\mathbb{R}^{N}} \int_{\mathbb{R}^{N}} \chi_{A^{\star}}(x) \chi_{A^{\star}}(y) \chi_{A^{\star}}(x-y) \mathrm{d} x \mathrm{~d} y .
\end{aligned}
$$

The first and the last term of the inequality are equal. By the work of Burchard on the necessary conditions for equality in the Riesz-Sobolev inequality, $A$ is an ellipsoid centered around the origin up to a set of measure zero [7].

Proposition 4.2. If $\cdot{ }^{*}$ is an anisotropic symmetrization and the inequality (4.1) holds for any $u, v, w \in \mathcal{K}_{+}\left(\mathbb{R}^{N}\right)$, then $G$ is an Euclidian norm on $\mathbb{R}^{k}$.

Proof. By standard arguments, the inequality (4.1) holds also for characteristic functions of open sets. Lemma 4.1 with $A=\mathrm{B}(0,1)^{*}$ brings the conclusion.

The same arguments shows also that the Riesz-Sobolev rearrangement inequality does not hold for the spherical cap symmetrization and for the polarization (see $[4,23,29]$ for definitions).

Corollary 4.3. If $*^{*}$ denotes the spherical cap symmetrization or the polarization, the inequality (4.1) does not hold for any $u, v, w \in \mathcal{K}_{+}\left(\mathbb{R}^{N}\right)$.

Proof. Suppose by contradiction that the inequality (4.1) holds for any $u, v, w \in \mathcal{K}_{+}\left(\mathbb{R}^{N}\right)$. By standard arguments, the inequality (4.1) holds also for characteristic functions of open sets. By Lemma 4.1, the set $E^{*}$ should be an ellipsoid when $E$ is an ellipsoid. This is not the case: for the spherical cap symmetrization, take e.g. the ellipsoid $\left\{x \in \mathbb{R}^{N}: \sum_{i=1}^{N} i x_{i}^{2}<1\right\}$, and for a polarization take an ellipsoid centered on the polarization plane and which is not symmetric with respect to it. This is possible for $N>1$.

If $N=1$ and the boundary of the polarizing halfspace is $\{c\}$, then the inequality fails for $u=\chi_{[2 c-1,2 c+1], v=}$ $\chi[-c-1,-c+1]$ and $w=\chi_{[c-1, c+1]}$.

The Riesz-Sobolev rearrangement inequality is a strong inequality, which requires good properties with respect to the convolution product. For the spherical cap symmetrization or the polarization the weaker inequality

$$
\iint_{\mathbb{R}^{N} \mathbb{R}^{N}} u(x) v(y) w(|x-y|) \mathrm{d} x \mathrm{~d} y \leqslant \iint_{\mathbb{R}^{N}} \int_{\mathbb{R}^{N}} u^{*}(x) v^{*}(y) w(|x-y|) \mathrm{d} x \mathrm{~d} y
$$


holds for any $u, v \in \mathcal{K}\left(\mathbb{R}^{N}\right)$ and for any decreasing function $w: \mathbb{R}^{+} \rightarrow \mathbb{R}^{+}[4,6,29]$. This is not the case for nontrivial anisotropic symmetrizations.

Proposition 4.4. Let $G: \mathbb{R}^{k} \rightarrow \mathbb{R}^{+}$be a gauge, and ${ }^{*}$ be the anisotropic symmetrization with respect to $G$. Let $\mu$ be a nonnegative Radon measure such that $\int_{\mathbb{R}^{N}}|x|_{2}^{2} \mathrm{~d} \mu<+\infty$. If the inequality

$$
\iint_{\mathbb{R}^{N}} u(x-y) v(y) \mathrm{d} \mu(x) \mathrm{d} y \leqslant \iint_{\mathbb{R}^{N}} \int_{\mathbb{R}^{N}} u^{*}(x-y) v^{*}(y) \mathrm{d} \mu(x) \mathrm{d} y .
$$

holds for any $u, v \in \mathcal{K}_{+}\left(\mathbb{R}^{N}\right)$, then either $\mu$ is concentrated on $\mathbb{R}^{k} \times\{0\}$ or $G\left(x^{\prime}\right)=\sqrt{x^{\prime t} A x^{\prime}}$ for some positive definite symmetric matrix $A \in \mathbb{R}^{k \times k}$. Furthermore, for any $u \in \mathcal{K}_{+}\left(\mathbb{R}^{N}\right)$,

$$
\int_{\mathbb{R}^{N}} u \mathrm{~d} \mu \leqslant \int_{\mathbb{R}^{N}} u^{*} \mathrm{~d} \mu .
$$

If $k=N$, then $\mu=w^{*}+a \delta_{0}$, where $w \in L_{+}^{1}\left(\mathbb{R}^{N}\right), a \geqslant 0$ and $\delta_{0}$ is Dirac's measure.

Remark 4.5. If $\mu=w \in L_{+}^{1}\left(\mathbb{R}^{N}\right)$, the condition (4.3) and Lemma 3.1 ensure $w=w^{*}$.

Remark 4.6. The inequality (4.2) always hold for measures concentrated on $T$. This is a consequence of the HardyLittlewood inequality (Proposition 2.28).

Proof. First suppose $k=N$. Without loss of generality, $\mu\left(\mathbb{R}^{N}\right)=1$. If (4.2) holds for any $u, v \in \mathcal{K}_{+}\left(\mathbb{R}^{N}\right)$, by density it holds also for any $u \in L_{+}^{1}\left(\mathbb{R}^{N}\right)$ and for any $v \in \mathcal{K}_{+}\left(\mathbb{R}^{N}\right)$. Take $u_{1} \in \mathcal{K}_{+}\left(\mathbb{R}^{N}\right)$ such that

$$
\int_{\mathbb{R}^{N}} u_{1} \mathrm{~d} x=1, \quad \int_{\mathbb{R}^{N}} u_{1}|x|_{2}^{2} \mathrm{~d} x<\infty, \quad \text { and } \quad u_{1}^{*}=u_{1} .
$$

For $n \geqslant 1$, let $u_{n+1}(x)=\int_{\mathbb{R}^{N}} u_{n}(x-y) \mathrm{d} \mu(y)$. By Fubini's theorem, $\int_{\mathbb{R}^{N}} u_{n+1} \mathrm{~d} x=1$. Furthermore, since $u_{n} \in$ $L^{1}\left(\mathbb{R}^{N}\right)$, inequality (4.2) holds with $u_{n}$ in place of $u$, and then, by Lemma $3.3, u_{n+1}{ }^{*}=u_{n+1}$. Let

$$
\bar{x}=\int_{\mathbb{R}^{N}} x \mathrm{~d} \mu .
$$

Consider the sequence of independent identically distributed random variables $\left(X_{n}\right)_{n} \geqslant 2$ with probability law $\mu$ and the independent random variable $X_{1}$ with distribution law $u_{1}$. All $X_{i}$ have mean $\bar{x}$ for $i \geqslant 2$. The function $u_{n}$ is the probability distribution of $\sum_{i=1}^{n} X_{i}$. By Lindeberg and Lévy's central-limit theorem (see Stromberg [24]), the sequence $n^{-1 / 2} \sum_{i=1}^{n}\left(X_{i}-\bar{x}\right)$ converges in law to a normal distribution $v$ with mean 0 , i.e. for any bounded continuous function $\varphi: \mathbb{R}^{N} \rightarrow \mathbb{R}$

$$
\int_{\mathbb{R}^{N}} \varphi\left(n^{-1 / 2}(x-n \bar{x})\right) u_{n}(y) \mathrm{d} x \rightarrow \int_{\mathbb{R}^{N}} \varphi \mathrm{d} \nu,
$$

where $v$ is characterized by

$$
\int_{\mathbb{R}^{N}} \varphi(x) \mathrm{d} v=(2 \pi)^{-N / 2} \int_{\mathbb{R}^{N}} \varphi(M x) \mathrm{e}^{-x^{2} / 2} \mathrm{~d} x
$$

for some fixed linear operator $M: \mathbb{R}^{N} \rightarrow \mathbb{R}^{N}$. (When $M$ is the identity one recovers the standard normal distribution. The operator $M$ is not necessarily invertible.)

For every $\varphi \in \mathcal{K}_{+}\left(\mathbb{R}^{N}\right)$, since $u_{n}^{*}=u_{n}$, one has

$$
\int_{\mathbb{R}^{N}} \varphi\left(n^{-1 / 2}(x-n \bar{x})\right) u_{n}(y) \mathrm{d} x \leqslant \int_{\mathbb{R}^{N}} \varphi^{*}\left(n^{-1 / 2} x\right) u_{n}(y) \mathrm{d} x .
$$


Since $v\left(\mathbb{R}^{N}\right)=1$, there is $\varphi \in \mathcal{K}_{+}\left(\mathbb{R}^{N}\right)$ such that $\varphi \leqslant 1$ and

$$
\int_{\mathbb{R}^{N}} \varphi \mathrm{d} v>\frac{1}{2}
$$

Therefore, for large $n$ both sides of the inequality (4.5) must be strictly greater than $1 / 2$. Since

$$
\int_{\mathbb{R}^{N}} u_{n} \mathrm{~d} x=1,
$$

this implies that the supports of $\varphi\left(n^{-1 / 2}(\cdot-n \bar{x})\right)$ and $\varphi\left(n^{-1 / 2}\right)$ have a nonempty intersection for large $n$. This is only possible if $\bar{x}=0$.

Since $\bar{x}=0$, letting $n \rightarrow \infty$ in (4.5) yields, by (4.4),

$$
\int_{\mathbb{R}^{N}} \varphi \mathrm{d} \nu \leqslant \int_{\mathbb{R}^{N}} \varphi^{*} \mathrm{~d} \nu .
$$

In view of Lemma 3.3, either the normal distribution $v$ is concentrated at zero, or $v=w \in L_{+}^{1}\left(\mathbb{R}^{N}\right)$, with $w(x)=$ $\mathrm{e}^{-x^{t} A x}, w=w^{*}$ and $A \in \mathbb{R}^{N \times N}$ is a positive-definite symmetric matrix. In the first case, this implies that

$$
\int_{\mathbb{R}^{N}}|x|_{2}^{2} \mathrm{~d} \mu=\int_{\mathbb{R}^{N}}|x|_{2}^{2} \mathrm{~d} \nu=0,
$$

and thus that the support of $\mu$ lies in $T$. In the second case, $w=w^{*}$ means $G(x)=\lambda \sqrt{x^{t} A x}$ for some $\lambda>0$.

Now, if $k<N$, let $\bar{\mu}$ denote the projection of $\mu$ on $\mathbb{R}^{k}$, i.e., if $\varphi \in \mathcal{K}\left(\mathbb{R}^{k}\right), \int_{\mathbb{R}^{k}} \varphi \mathrm{d} \bar{\mu}=\int_{\mathbb{R}^{N}} \varphi\left(x^{\prime}\right) \mathrm{d} \mu$. Then the inequality (4.2) holds for $\bar{\mu}$. By the first part of the proof, either $G$ is Euclidian or $\bar{\mu}$ is concentrated at 0 , whence $\mu$ is concentrated on $\{0\} \times \mathbb{R}^{k}=T$.

For (4.3), take $\rho \in \mathcal{K}_{+}\left(\mathbb{R}^{N}\right)$ with $\int_{\mathbb{R}^{N}} \rho \mathrm{d} x=1$ and $\rho_{\varepsilon}=\varepsilon^{-N} \rho(\dot{\bar{\varepsilon}})$. Inequality (4.2) with $v=\rho_{\varepsilon}$ gives (4.3) as $\varepsilon \rightarrow 0$.

The Riesz-Sobolev type inequalities (4.1) and (4.2) are useful to prove Pólya-Szegó inequalities (see [4,19]). Propositions 4.2 and 4.4 show that this is not a valid method for the anisotropic symmetrization.

\section{Anisotropic inequalities for Steiner symmetrizations}

The objective of this section is to prove that for any Steiner symmetrization $\cdot^{\star}$, any suitable weakly differentiable $u$ and any function $\varphi: \mathbb{R}^{N} \rightarrow \overline{\mathbb{R}}^{+}$such that $\varphi(0)=0$, the inequality

$$
\int_{\mathbb{R}^{N}} \overline{\varphi_{\star}}\left(\nabla u^{\star}\right) \mathrm{d} x \leqslant \int_{\mathbb{R}^{N}} \bar{\varphi}(\nabla u) \mathrm{d} x
$$

holds. Recall that $\varphi_{\star}$ denotes the increasing Steiner symmetrization of $\varphi$, i.e. $\varphi_{\star}=-\varphi(-\cdot)^{\star}$ (see Definition 2.33 and Proposition 2.34). Klimov proved this inequality for the Steiner symmetrization with respect to a hyperplane when $\varphi: \mathbb{R}^{N} \rightarrow \mathbb{R}$ is convex and even [18]. He suggested the inequality for a general Steiner symmetrization. We first prove the inequality for the Steiner symmetrization with respect to a hyperplane and then extend it to general Steiner symmetrizations.

Definition 5.1. The Fenchel transform of $\varphi: \mathbb{R}^{N} \rightarrow \overline{\mathbb{R}}$ is

$$
\bar{\varphi}: \mathbb{R}^{N} \rightarrow \overline{\mathbb{R}}: t \mapsto \bar{\varphi}(t)=\sup _{x \in \mathbb{R}^{N}}\langle t, x\rangle-\varphi(x) .
$$

Remark 5.2. By an abuse of notation, when $\varphi$ comes from a functional of the form

$$
\int_{\Omega} \varphi(x, u, \nabla u) \mathrm{d} x,
$$


then $\bar{\varphi}$ denotes the Fenchel transform and the symmetrization with respect to the gradient coordinates: $\bar{\varphi}(x, s, \cdot)=$ $\overline{\varphi(x, s, \cdot)}$. The same abuse of notation is made for the symmetrization: $\varphi_{\star}(x, s, \cdot)=(\varphi(x, s, \cdot))_{\star}$.

Proposition 5.3. Let $\varphi$ and $\psi$ be functions from $\mathbb{R}^{N}$ to $\overline{\mathbb{R}}$. If $\varphi \leqslant \psi$, then $\bar{\varphi} \geqslant \bar{\psi}$. Let $\left(\varphi_{n}\right)_{n} \geqslant 1$ and $\varphi$ be functions from $\mathbb{R}^{N}$ to $\overline{\mathbb{R}}$. If $\varphi_{n} \searrow \varphi$, then $\bar{\varphi}_{n} \nearrow \bar{\varphi}$.

Proof. Immediate.

Definition 5.4. An open set $\Omega$ is an extension domain if there exists a bounded linear operator $E_{\Omega}: W^{1,1}(\Omega) \rightarrow$ $W^{1,1}\left(\mathbb{R}^{N}\right)$, such that, for each $u \in W^{1,1}(\Omega),\left.\left(E_{\Omega} u\right)\right|_{\Omega}=u$.

Example 5.5. A Lipschitz domain is an extension domain [1].

Proposition 5.6 ((1-dimensional Steiner symmetrization inequality for anisotropic functionals)). Let $\varphi: \mathbb{R}^{N} \rightarrow \overline{\mathbb{R}}^{+}$ with $\varphi(0)=0$. If $T$ is a $(N-1)$-dimensional vector subspace of $\mathbb{R}^{N}, .^{\star}$ is the Steiner symmetrization with respect to $T, \Omega$ is an extension domain, $\Omega$ is totally invariant with respect to $T$ and $u \in W_{+}^{1,1}\left(\mathbb{R}^{N}\right)$, then

$$
\int_{\Omega} \overline{\varphi_{\star}}\left(\nabla u^{\star}\right) \mathrm{d} x \leqslant \int_{\Omega} \bar{\varphi}(\nabla u) \mathrm{d} x .
$$

Remark 5.7. The hypothesis $\varphi(0)=0$ ensures $\bar{\varphi}(t) \geqslant 0$ for $t \in \mathbb{R}^{N}$, while $\varphi \geqslant 0$ implies $\bar{\varphi}(0) \leqslant 0$.

Definition 5.8. A function $u: \Omega \rightarrow \mathbb{R}$ is simplicial if it is continuous, it has a bounded support, and if there exists a finite collection of open sets $\left(S_{i}\right)_{1 \leqslant i \leqslant n}$, such that $\left.\tilde{u}\right|_{S_{i}}$ is an affine function for each $1 \leqslant i \leqslant n$ and $u$ vanishes outside the closure of $\bigcup_{i=1}^{n} S_{i}$.

It is standard that if $\Omega$ is an extension domain, then simplicial functions are dense in $W^{1,1}(\Omega)$ [13, Chapter X, Section 2.1]. Furthermore, simplicial functions with $\frac{\partial u}{\partial x_{1}} \neq 0$ in $\bigcup_{i=1}^{n} S_{i}$ are also dense in $W^{1,1}(\Omega)$.

Proof of Proposition 5.6. The proof is an adaptation from Klimov [18], with modifications allowing more general functions $\varphi$ and other domains than $\mathbb{R}^{N}$. For simple functions, it relies on the geometric results in Lemma 5.12 and a coarea formula. The result is extended by density to non-simplicial functions with some restriction on $\varphi$ and is finally generalized to any function $\varphi$ by Levi's monotone convergence theorem.

Step 1: $u$ is a simplicial function. Without loss of generality, let $T=\{0\} \times \mathbb{R}^{N-1}$, and $\Omega=\mathbb{R} \times \Omega^{\prime \prime}$. Suppose $u \in W^{1,1}(\Omega)$ is a nonnegative simplicial function such that $\partial_{1} u \neq 0$ on $\bigcup_{i=1}^{n} S_{i}$. On each set $S_{i}, \nabla u$ is constant. We have thus

$$
\begin{aligned}
\int_{S_{i}} \bar{\varphi}(\nabla u) \mathrm{d} x & =\int_{\Omega^{\prime \prime}} \int_{\left\{x_{1} \in \mathbb{R}:\right.} \overline{\left.\varphi\left(x_{1}, x^{\prime \prime}\right) \in S_{i}\right\}} \frac{\bar{\varphi}(\nabla u) \mathrm{d} x_{1} \mathrm{~d} x^{\prime \prime}}{} \\
& =\int_{\Omega^{\prime \prime}\left\{s>0:\left(\left.u\right|_{S_{i} \cap\left\{\left(x^{\prime \prime}, t\right): t \in \mathbb{R}\right\}}\right)^{-1}(\{s\}) \neq \phi\right\}} \frac{\bar{\varphi}(\nabla u)}{\left|\partial_{1} u\right|} \mathrm{d} s \mathrm{~d} x^{\prime \prime} \\
& =\int_{\Omega^{\prime \prime}} \int_{s>0} \sum_{\left(x_{1}, x^{\prime \prime}\right) \in\left(u^{-1}(\{s\}) \cap S_{i}\right)} \frac{\bar{\varphi}\left(\nabla u\left(x_{1}, x^{\prime \prime}\right)\right)}{\left|\partial_{1} u\left(x_{1}, x^{\prime \prime}\right)\right|} \mathrm{d} s \mathrm{~d} x_{1},
\end{aligned}
$$

where the sum contains zero or one term. Summing over $i$ gives, since $\nabla u(x)=0$ for almost all $x \notin \bigcup_{i=1}^{n} S_{i}$, and $\bar{\varphi}(0)=0$ by Remark 5.7,

$$
\int_{\Omega} \bar{\varphi}(\nabla u) \mathrm{d} x=\int_{\Omega^{\prime \prime}} \int_{s>0} \sum_{\left(x_{1}, x^{\prime \prime}\right) \in u^{-1}(\{s\})} \frac{\bar{\varphi}\left(\nabla u\left(x_{1}, x^{\prime \prime}\right)\right)}{\left|\partial_{1} u\left(x_{1}, x^{\prime \prime}\right)\right|} \mathrm{d} s \mathrm{~d} x^{\prime \prime} .
$$


For each $x^{\prime \prime} \in \Omega^{\prime \prime}$, the sum contains always a finite number of terms (at most $n$ ). Furthermore, the number of terms for which $\partial_{1} u\left(x_{1}, x^{\prime \prime}\right)>0$ is equal to the number of terms for which $\partial_{1} u\left(x_{1}, x^{\prime \prime}\right)<0$. Similarly,

$$
\begin{aligned}
\int_{\Omega} \overline{\varphi_{\star}}\left(\nabla u^{\star}\right) \mathrm{d} x & =\int_{\Omega^{\prime \prime}} \int_{s>0} \sum_{\left(x_{1}, x^{\prime \prime}\right) \in u^{-1}(s)} \frac{\overline{\varphi_{\star}}\left(\nabla u^{\star}\left(x_{1}, x^{\prime \prime}\right)\right)}{\left|\partial_{1} u^{\star}\left(x_{1}, x^{\prime \prime}\right)\right|} \mathrm{d} s \mathrm{~d} x^{\prime \prime} \\
& =2 \int_{\Omega^{\prime \prime}} \int_{s>0} \frac{\overline{\varphi_{\star}}\left(\nabla u^{\star}\right)}{\left|\partial_{1} u^{\star}\right|}\left(\left(\left.u^{\star}\right|_{\Omega^{\prime \prime} \times \mathbb{R}^{+}}\right)^{-1}(\{s\})\right) \mathrm{d} s \mathrm{~d} x^{\prime \prime} .
\end{aligned}
$$

For all $x^{\prime \prime} \in \Omega^{\prime \prime}$, the definition of the Steiner symmetrization gives, for all but a finite number of $s \in\left(0, \sup u\left(\cdot, x^{\prime \prime}\right)\right)$,

$$
\begin{aligned}
& 2 \frac{\nabla^{\prime \prime} u^{\star}}{\left|\partial_{1} u^{\star}\right|}\left(\left(\left.u^{\star}\right|_{\mathbb{R}^{+} \times\left\{x^{\prime \prime}\right\}}\right)^{-1}(s)\right)=\sum_{\left(x_{1}, x^{\prime \prime}\right) \in u^{-1}(s)} \frac{\nabla^{\prime \prime} u\left(x_{1}, x^{\prime \prime}\right)}{\left|\partial_{1} u\left(x_{1}, x^{\prime \prime}\right)\right|}, \\
& 2 \frac{1}{\left|\partial_{1} u^{\star}\right|}\left(\left(\left.u^{\star}\right|_{\mathbb{R}^{+} \times\left\{x^{\prime \prime}\right\}}\right)^{-1}(s)\right)=\sum_{\left(x_{1}, x^{\prime \prime}\right) \in u^{-1}(s)} \frac{1}{\left|\partial_{1} u\left(x_{1}, x^{\prime \prime}\right)\right|} .
\end{aligned}
$$

Then by Lemma 5.12, inequality (5.1) holds for $u$.

Step 2: extension to $u \in W_{+}^{1,1}(\Omega)$. Suppose $u \in W_{+}^{1,1}(\Omega)$ and that there exists $R \in \mathbb{R}$ such that $\varphi(x)=\infty$ if $|x|_{2}>R$. Then $\bar{\varphi}(t) \leqslant|t|_{2} / R$ and $\overline{\varphi_{\star}}(t) \leqslant|t|_{2} / R$ (since the right-hand side of the inequality is symmetrizationinvariant). The functionals on both sides of $(5.1)$ are continuous in $W^{1,1}(\Omega)$. Since simplicial functions are dense in $W^{1,1}(\Omega)$ and the Steiner symmetrization is continuous in $W^{1,1}(\Omega)$ [8], the inequality follows.

(Alternatively, the nonexpansiveness of symmetrization in $L^{1}(\Omega)$ and the classical Pólya-Szegó inequality can be used to prove that if $u_{n} \rightarrow u$ in $W^{1,1}(\Omega)$, then $u_{n}^{\star} \rightarrow u^{\star}$ in $W^{1,1}(\Omega)$. The inequality comes then from the weak lower semi-continuity of the left-hand side with respect to $u \in W^{1,1}(\Omega)$.)

Step 3: general $\varphi$. For general $u \in W_{+}^{1,1}(\Omega)$ and $\varphi: \mathbb{R}^{N} \rightarrow \overline{\mathbb{R}}^{+}$, let

$$
\varphi_{n}(x)= \begin{cases}\varphi(x) & \text { if }|x|_{2} \leqslant n, \\ +\infty & \text { if }|x|_{2}>n\end{cases}
$$

for $n \geqslant 1$. Since $\varphi_{n} \searrow \varphi$ for $x \in \Omega$, Propositions 2.20 and 5.3 imply $\varphi_{n \star} \searrow \varphi_{\star}, \overline{\varphi_{n}} \nearrow \bar{\varphi}$ and $\overline{\varphi_{n \star}} \nearrow \overline{\varphi_{\star}}$. The inequality follows by Levi's monotone convergence theorem.

We now prove Klimov's geometric lemma used in the proof of the inequality for the 1-dimensional Steiner symmetrization. The simplest case is when $\varphi$ is the indicator function of some set $A$ :

$$
\varphi(x)= \begin{cases}0 & \text { if } x \in A, \\ +\infty & \text { if } x \notin A .\end{cases}
$$

Definition 5.9. The function

$$
\delta_{A}(t)=\bar{\varphi}(t)=\sup _{x \in A}\langle t, x\rangle
$$

is the support function of $A$.

Lemma 5.10. Let $\cdot^{\star}$ be the Steiner symmetrization with respect to $\{0\} \times \mathbb{R}^{N-1}$ and $A \subseteq \mathbb{R}^{N}$. For any a, $b \in \mathbb{R}^{N-1}$,

$$
\delta_{A}(1, a)+\delta_{A}(-1, b) \geqslant \delta_{A^{\star}}(2, a+b) .
$$

Remark 5.11. Klimov proves this result for a convex compact set $A$. His representation of the set $A=\left\{\left(x_{1}, x^{\prime \prime}\right)\right.$ : $\left.h\left(x^{\prime \prime}\right) \leqslant x_{1} \leqslant g\left(x^{\prime \prime}\right)\right\}$ is not anymore valid, but his arguments can be adapted to sets which are not bounded, measurable and convex. 
Proof. For $x^{\prime \prime} \in \mathbb{R}^{N-1}$, let

$$
\begin{aligned}
& m_{A}\left(x^{\prime \prime}\right)=\inf \left\{x_{1}:\left(x_{1}, x^{\prime \prime}\right) \in A\right\}, \\
& M_{A}\left(x^{\prime \prime}\right)=\sup \left\{x_{1}:\left(x_{1}, x^{\prime \prime}\right) \in A\right\} .
\end{aligned}
$$

Then

$$
\begin{aligned}
& \delta_{A}(1, a)=\sup _{x^{\prime \prime} \in \mathbb{R}^{N-1}}\left\langle a, x^{\prime \prime}\right\rangle+M_{A}\left(x^{\prime \prime}\right), \\
& \delta_{A}(-1, b)=\sup _{x^{\prime \prime} \in \mathbb{R}^{N-1}}\left\langle b, x^{\prime \prime}\right\rangle-m_{A}\left(x^{\prime \prime}\right) .
\end{aligned}
$$

The identities hold with $A^{\star}$ in place of $A$. The inequality comes from the fact that for all $x^{\prime \prime} \in \mathbb{R}^{N-1}, 2 M_{A^{\star}}\left(x^{\prime \prime}\right)=$ $2 m_{A^{\star}}\left(x^{\prime \prime}\right) \leqslant M_{A}\left(x^{\prime \prime}\right)-m_{A}\left(x^{\prime \prime}\right)$ by definition of the Steiner symmetrization.

Lemma 5.12 ((Klimov)). Let $\varphi: \mathbb{R}^{N} \rightarrow \overline{\mathbb{R}}, a_{i}, b_{i} \in \mathbb{R}^{N-1}, \alpha_{i}, \beta_{i} \in \mathbb{R}^{+}$, for all $1 \leqslant i \leqslant m$. If $\alpha=\sum_{i=1}^{m} \alpha_{i}, \beta=$ $\sum_{i=1}^{m} \beta_{i}, a=\sum_{i=1}^{m} a_{i}$ and $b=\sum_{i=1}^{m} b_{i}$, then

$$
\sum_{i=1}^{m} \bar{\varphi}\left(\frac{1}{\alpha_{i}}, \frac{a_{i}}{\alpha_{i}}\right) \alpha_{i}+\bar{\varphi}\left(-\frac{1}{\beta_{i}}, \frac{b_{i}}{\beta_{i}}\right) \beta_{i} \geqslant \overline{\varphi_{\star}}\left(\frac{2}{\alpha+\beta}, \frac{a+b}{\alpha+\beta}\right)(\alpha+\beta) .
$$

Proof. Without loss of generality, the left-hand side of (5.2) is finite. By definition of the Fenchel transform,

$$
\bar{\varphi}(t)=\sup _{x \in \mathbb{R}^{N}}\langle t, x\rangle-\varphi(x)=\sup _{\substack{x \in\{\varphi<\lambda\} \\ \lambda \in \mathbb{R}}}\langle t, x\rangle-\lambda=\sup _{\lambda \in \mathbb{R}}\left[\sup _{x \in\{\varphi<\lambda\}}\langle t, x\rangle-\lambda\right]=\sup _{\lambda \in \mathbb{R}}\left(\delta_{\{\varphi<\lambda\}}(t)-\lambda\right) .
$$

Thus for any $\lambda \in \mathbb{R}$,

$$
\begin{aligned}
\sum_{i=1}^{m}\left[\bar{\varphi}\left(\frac{1}{\alpha_{i}}, \frac{a_{i}}{\alpha_{i}}\right) \alpha_{i}+\bar{\varphi}\left(-\frac{1}{\beta_{i}}, \frac{b_{i}}{\beta_{i}}\right) \beta_{i}\right] & \geqslant \sum_{i=1}^{m}\left[\delta_{\{\varphi<\lambda\}}\left(\frac{1}{\alpha_{i}}, \frac{a_{i}}{\alpha_{i}}\right)-\lambda\right] \alpha_{i}+\left[\delta_{\{\varphi<\lambda\}}\left(-\frac{1}{\beta_{i}}, \frac{b_{i}}{\beta_{i}}\right)-\lambda\right] \beta_{i} \\
& =\left[\sum_{i=1}^{m} \delta_{\{\varphi<\lambda\}}\left(1, a_{i}\right)+\delta_{\{\varphi<\lambda\}}\left(-1, b_{i}\right)\right]-\lambda(\alpha+\beta) .
\end{aligned}
$$

By Lemma 5.10, and since the function $\delta_{\{\varphi \leqslant \lambda\}^{\star}}\left(t_{1}, t^{\prime \prime}\right)$ increases with respect to $t_{1}$ when $t_{1} \geqslant 0$,

$$
\sum_{i=1}^{m}\left[\bar{\varphi}\left(\frac{1}{\alpha_{i}}, \frac{a_{i}}{\alpha_{i}}\right) \alpha_{i}+\bar{\varphi}\left(-\frac{1}{\beta_{i}}, \frac{b_{i}}{\beta_{i}}\right) \beta_{i}\right] \geqslant \delta_{\{\varphi<\lambda\}^{\star}}(2 m, a+b)-\lambda(\alpha+\beta) \geqslant \delta_{\{\varphi<\lambda\}^{\star}}(2, a+b)-\lambda(\alpha+\beta) .
$$

This implies the inequality since, from the first part of the proof and from Proposition 2.20,

$$
\overline{\varphi_{\star}}(t)=\sup _{\lambda \in \mathbb{R}}\left[\delta_{\left\{\varphi_{\star}<\lambda\right\}}(t)-\lambda\right]=\sup _{\lambda \in \mathbb{R}}\left[\delta_{\{\varphi<\lambda\}^{\star}}(t)-\lambda\right] .
$$

We extend the inequality to Steiner symmetrizations with respect to higher dimensional subspaces by approximation, as suggested by Klimov [18]. Since the approximation procedure in Hausdorff distance of open sublevel sets is unusual, we give a complete proof.

Proposition 5.13 ((Steiner symmetrization inequality for anisotropic functionals)). Let $T \subset \mathbb{R}^{N}$ be a vector space, $\Omega$ a totally invariant extension domain, . ${ }^{\star}$ denote the Steiner symmetrization with respect to $T, u \in W_{+}^{1,1}(\Omega)$ and $\varphi: \mathbb{R}^{N} \rightarrow \overline{\mathbb{R}}^{+}$such that $\varphi(0)=0$. If $u$ vanishes at the infinity with respect to $\cdot^{\star}$, and for any $M>0$,

$$
\sup _{\mathcal{L}^{N}(A)=M} \int_{A}|\nabla u|_{2}+|u| \mathrm{d} x<+\infty
$$


then $u^{\star} \in W_{\mathrm{loc}}^{1,1}(\Omega)$ and

$$
\int_{\Omega} \overline{\varphi_{\star}}\left(\nabla u^{\star}\right) \mathrm{d} x \leqslant \int_{\Omega} \bar{\varphi}(\nabla u) \mathrm{d} x
$$

Remark 5.14. In general, when $u \in W_{\mathrm{loc}}^{1,1}(\Omega)$, it is not true that $u^{*} \in W_{\mathrm{loc}}^{1,1}(\Omega)$. The condition (5.4) is a slightly stronger than $u \in W_{\mathrm{loc}}^{1,1}(\Omega)$ and guarantees that $u^{*} \in W_{\mathrm{loc}}^{1,1}(\Omega)$.

Proof. The inequality is established by approximation of the symmetrization for $u \in W_{+}^{1,1}(\Omega)$ and $\varphi$ with a finite image, and then extended to general $\varphi$ and then general $u$.

Step 1: $\varphi$ has a finite image. First suppose $u \in W_{+}^{1,1}(\Omega), \varphi$ is lower semi-continuous and coercive, and $\varphi$ has a finite image, i.e. the set $\varphi\left(\mathbb{R}^{N}\right)$ is finite. The conclusion is contained in Proposition 5.6 if $\operatorname{dim} T=N-1$. From now on, $\operatorname{dim} T<N-1$. By classical approximation results in symmetrization theory $[5,6,19,28]$, there exists a sequence of $(N-1)$-dimensional hyperplanes $\left(T_{n}\right)_{n} \geqslant 1$ such that, if $\cdot T_{n}$ denotes the Steiner symmetrization with respect to $T_{n}$,

(i) for any $n \geqslant 1, T \subset T_{n}$,

(ii) the iterated sequence of symmetrizations $u_{n}=u^{T_{1} \cdots T_{n}}$ converges to $u^{\star}$ :

$$
\begin{array}{ll}
u_{n} \rightarrow u^{\star} & \text { in } W^{1,1}(\Omega), \\
u_{n} \rightarrow u^{\star} & \text { in } L^{1}(\Omega) ;
\end{array}
$$

(iii) if $A$ is measurable and $A^{\star}$ is bounded and open, then the sequence of iterated symmetrizations $A_{n}=A^{T_{1} \cdots T_{n}}$, converges to $A^{\star}$ in the sense that small neighborhoods of $A_{n}$ contain $A^{\star}$ :

$$
\lim _{n \rightarrow \infty} \sup _{x \in A^{\star}} \mathrm{d}\left(x, A_{n}\right)=0 .
$$

This last assertion is proved in Lemma 5.16 .

Since the function $\varphi$ is lower semi-continuous and $\lim _{|t|_{2} \rightarrow \infty} \varphi(t)=+\infty$, the set $\{\varphi<\lambda\}$ is open and bounded for each $\lambda \geqslant 0$. Hence the set $\{\varphi<\lambda\}^{*}$ is open and bounded. Since $\varphi$ has a finite image, the convergence of sublevel sets is uniform with respect to levels in (5.6):

$$
\lim _{n \rightarrow \infty} \sup _{\substack{\lambda>0 \\ x \in\left\{\varphi_{\star}<\lambda\right\}}} \mathrm{d}\left(x,\{\varphi<\lambda\}^{T_{1} \cdots T_{n}}\right)=0 .
$$

Thus for any $\varepsilon>0$, there exists $n_{0} \geqslant 0$ such that for all $n \geqslant n_{0}, x \in \mathbb{R}^{N}$, there exists $y \in \mathbb{R}^{N}$ such that $|x-y|_{2} \leqslant \varepsilon$ and $\varphi_{\star}(x) \geqslant \varphi_{n}(y)=\varphi_{T_{1} \cdots T_{n}}(y)$. Therefore

$$
\overline{\varphi_{\star}}(t)=\sup _{x \in \mathbb{R}^{N}}\langle t, x\rangle-\varphi_{\star}(x) \leqslant \varepsilon|t|_{2}+\sup _{y \in \mathbb{R}^{N}}\langle t, y\rangle-\varphi_{n}(y)=\varepsilon|t|_{2}+\overline{\varphi_{n}}(t) .
$$

and

$$
\int_{\Omega} \overline{\varphi_{\star}}\left(\nabla u_{n}\right) \leqslant \varepsilon\left\|\nabla u_{n}\right\|_{1}+\int_{\Omega} \overline{\varphi_{n}}\left(\nabla u_{n}\right) .
$$

Since $\overline{\varphi_{\star}}$ is convex and lower semi-continuous in $\mathbb{R}^{N}$, the left-hand side is lower semi-continuous in $W^{1,1}(\Omega)$. By induction on Proposition 5.6 and by letting $n \rightarrow \infty$,

$$
\int_{\Omega} \overline{\varphi_{\star}}\left(\nabla u^{\star}\right) \mathrm{d} x \leqslant \liminf _{m \rightarrow \infty} \int_{\Omega} \overline{\varphi_{\star}}\left(\nabla u_{n}\right) \mathrm{d} x \leqslant \liminf _{m \rightarrow \infty} \varepsilon\left\|\nabla u_{n}\right\|_{1}+\liminf _{m \rightarrow \infty} \int_{\Omega} \overline{\varphi_{n}}\left(\nabla u_{n}\right) \mathrm{d} x \leqslant \varepsilon\|\nabla u\|_{1}+\int_{\Omega} \bar{\varphi}(\nabla u) \mathrm{d} x .
$$

Since $\varepsilon>0$ is arbitrary, the result follows. 
Step 2: general $\varphi$. If $\varphi$ does not have a finite image or is not coercive, but is lower semi-continuous, then it can be approximated by a decreasing sequence of coercive and lower semi-continuous functions with a finite image $\varphi_{n} \searrow \varphi$. Because $\overline{\varphi_{n}} \nearrow \bar{\varphi}$, the result follows by Levi's monotone convergence theorem. If $\varphi$ is not lower semi-continuous, let $\psi(x)=\liminf _{y \rightarrow x} \varphi(y)$. Then $\bar{\psi}=\bar{\varphi}$ and $\overline{\psi_{\star}} \geqslant \overline{\varphi_{\star}}$, whence the inequality for $\varphi$ follows from the inequality for $\psi$.

Step 3: general $u$. Let $u_{m}=\max (u-1 / m, 0)$. It is clear that $u_{m}$ converges uniformly to $u$, so that $u_{m}^{\star}$ converges to $u^{\star}$ uniformly and in $L_{\text {loc }}^{1}(\Omega)$. Furthermore $u_{m}$ converges to $u$ in $W_{\text {loc }}^{1,1}(\Omega)$ by Lebesgue's dominated convergence theorem. The sequence of functions $\left|\nabla u_{m}^{\star}\right|_{2}$ in $L_{\text {loc }}^{1}(\Omega)$ is also nondecreasing. By a result of Alvino, Ferone and Lions [3], for any compact subset $K$ of $\Omega$,

$$
\int_{K}\left|\nabla u_{m}^{\star}\right|_{2} \leqslant \sup _{\mathcal{L}^{N}(L)=\mathcal{L}^{N}(K)} \int_{L}\left|\nabla u_{m}\right|_{2} .
$$

Hence, if $g(x)=\lim _{n \rightarrow \infty} \nabla u_{m}(x)$, by Levi's monotone convergence theorem,

$$
\int_{K}|g|_{2}<+\infty
$$

This implies, by Lebesgue's dominated convergence theorem, that $\nabla u_{m}^{\star} \rightarrow g$ in $L_{\text {loc }}^{1}(\Omega)$. Thus $u^{\star} \in W_{\text {loc }}^{1,1}(\Omega)$. The inequality follows by Levi's monotone convergence theorem.

We have to prove the convergence result of iterated symmetrizations of sets. Since our definition of symmetrization is different from the classical one that maps compact sets into compact sets, we cannot use the classical results on approximation in Hausdorff distance. Lemma 5.15 is a general measure-theoretic convergence result.

Lemma 5.15. Suppose $\left(A_{n}\right)_{n} \geqslant 1$ is a sequence of measurable sets in $\mathbb{R}^{N}, G \subset \mathbb{R}^{N}$ is open, bounded, and nonempty. If

$$
\lim _{n \rightarrow \infty} \mathcal{L}^{N}\left(G \backslash A_{n}\right)=0,
$$

then

$$
\lim _{n \rightarrow \infty} \sup _{x \in G} \mathrm{~d}\left(x, A_{n}\right)=0,
$$

where

$$
\mathrm{d}(x, A)=\inf _{y \in A} \mathrm{~d}(x, y) .
$$

Proof. Suppose the conclusion is false. Then there exists an increasing sequence $\left(n_{k}\right)_{k \in \mathbb{N}}$ in $\mathbb{N}$, a sequence $x_{k} \in G$ and $\delta>0$ such that, for each $k \in \mathbb{N}$,

$$
\mathrm{d}\left(x_{k}, A_{n_{k}}\right)>\delta .
$$

Since $G$ is a bounded subset of $\mathbb{R}^{N}$, the sequence $\left(x_{k}\right)_{k \in \mathbb{N}}$ has a subsequence $\left(x_{k_{\ell}}\right)_{\ell \in \mathbb{N}}$ that converges to $\tilde{x} \in \bar{G}$. If $\ell$ is sufficiently large,

$$
\phi \neq \mathrm{B}\left(\tilde{x}, \frac{\delta}{2}\right) \cap G \subset \mathrm{B}\left(x_{k_{\ell}}, \delta\right) \cap G \subseteq G \backslash A_{n_{k_{\ell}}} .
$$

Because $\mathrm{B}\left(\tilde{x}, \frac{\delta}{2}\right) \cap G$ is open and not empty,

$$
\mathcal{L}^{N}\left(G \backslash A_{n_{k_{\ell}}}\right) \geqslant \mathcal{L}^{N}\left(\mathrm{~B}\left(\tilde{x}, \frac{\delta}{2}\right) \cap G\right)>0,
$$

in contradiction with (5.7). 
Lemma 5.16. Suppose $G \in \mathbb{R}^{N}$ is a bounded open set and $\left(G_{n}\right)_{n \geqslant 1}$ is the sequence of sets obtained by iterated Steiner symmetrizations of $G$. Then

$$
\lim _{n \rightarrow \infty} \sup _{x \in G^{\star}} \mathrm{d}\left(x, G_{n}\right)=0 .
$$

Proof. This comes from the fact that $G^{\star}$ is open, the convergence in measure of $G_{n}$ to $G^{\star}$ and Lemma 5.15.

Proposition 5.17. Let $\Omega$ be a totally invariant domain, $\varphi: \Omega \times \mathbb{R}^{+} \times \mathbb{R}^{N} \rightarrow \overline{\mathbb{R}}^{+}$and $u \in W_{\text {loc, }+}^{1,1}(\Omega)$ vanishing at the infinity with respect to ${ }^{*}$. If $\varphi(\cdot, s, \xi)$ is totally invariant with respect to $\cdot^{\star}$ for each $(s, \xi) \in \mathbb{R}^{+} \times \mathbb{R}^{N}, \varphi(\cdot, \cdot, 0)=0$, $\varphi(\cdot, \cdot, \xi)$ is lower semi-continuous for each $\xi \in \mathbb{R}^{N}$ and if each $x \in \Omega$ has a totally invariant neighborhood $\mathcal{N}_{x} \subset \Omega$ such that for any $M>0$,

$$
\sup _{\substack{A \subset \mathcal{N}_{x} \\ \mathcal{L}^{N}(A)=M}} \int_{A}|\nabla u|_{2}+|u| \mathrm{d} x<+\infty,
$$

then

$$
\int_{\Omega} \overline{\varphi_{\star}}\left(x, u^{\star}(x), \nabla u^{\star}(x)\right) \mathrm{d} x \leqslant \int_{\Omega} \bar{\varphi}(x, u(x), \nabla u(x)) \mathrm{d} x .
$$

Proof. Without loss of generality, $T=\mathbb{R}^{k}$ and $\Omega=\mathbb{R}^{N-k} \times \Omega^{\prime \prime}$. Let

$$
\begin{gathered}
\mathcal{B}_{m}=\left\{\beta=\mathbb{R}^{N-k} \times\left(\frac{\ell_{N-k+1}}{2^{m}}, \frac{\ell_{N-k+1}+1}{2^{m}}\right) \times \cdots \times\left(\frac{\ell_{N}}{2^{m}}, \frac{\ell_{N}+1}{2^{m}}\right) \times\left(\frac{n}{2^{m}}, \frac{n+1}{2^{m}}\right):\right. \\
\left.\ell_{i} \in \mathbb{Z}, n \in \mathbb{N}, \beta \subset \Omega \times \mathbb{R}^{+} \text {and } u \text { verifies (5.8) with } \mathcal{N}_{x}=P(\beta)\right\} .
\end{gathered}
$$

where $P$ is the projection $P: \Omega \times \mathbb{R}^{+} \rightarrow \Omega:(x, s) \mapsto x$. Let $\Omega_{m}=P\left(\bigcup_{\beta \in \mathcal{B}_{m}} \beta\right)$. For $(x, s) \in \Omega_{m} \times \mathbb{R}^{+}$, let $\beta_{m}(x)$ denote the unique $\beta \in \mathcal{B}_{m}$ such that $(x, s) \in \beta$. Let $\omega_{m}(x)=P\left(\beta_{m}(x)\right)$. For any $(x, s) \in \Omega_{m} \times \mathbb{R}^{+}$, let

$$
\varphi_{m}(x, s, \xi)=\sup _{(y, t) \in \beta_{m}(x, s)} \varphi(y, t, \xi) .
$$

Fix $\ell>0$. From Proposition 5.13, it is clear that for any $\omega_{m}(x) \subset \Omega_{m}$, and $u_{n, m}=\max \left(n / 2^{m}, \min \left(u,(n+1) / 2^{m}\right)\right)$,

$$
\int_{\omega_{m}(x)} \overline{\varphi_{m \star}}\left(x, u_{n, m}{ }^{\star}, \nabla u_{n, m}{ }^{\star}\right) \leqslant \int_{\omega_{m}(x)} \overline{\varphi_{m}}\left(x, u_{n, m}, \nabla u_{n, m}\right)
$$

since $\varphi_{m}(\cdot, \cdot, \xi)$ is constant on $\beta$ for any $\xi \in \mathbb{R}^{N}$. Since $\Omega_{\ell} \subset \Omega_{m}$ up to a set of measure zero, the sum with fixed $m$ for all $\omega_{m}(x)$ with $\omega_{m}(x) \subset \Omega_{\ell}$ and $n \geqslant 0$ is

$$
\int_{\Omega_{\ell}} \overline{\varphi_{m \star}}\left(x, u^{\star}, \nabla u^{\star}\right) \leqslant \int_{\Omega_{\ell}} \overline{\varphi_{m}}(x, u, \nabla u) .
$$

Since $\varphi(\cdot, \cdot, \xi)$ is lower semi-continuous, $\varphi_{m}(\cdot, \cdot, \xi) \searrow \varphi(\cdot, \cdot, \xi)$ as $k \rightarrow \infty$. Therefore $\overline{\varphi_{m}}(x, s, \cdot) \nearrow \bar{\varphi}(x, s, \cdot)$ and $\overline{\varphi_{m_{\star}}}(x, s, \cdot) \nearrow \overline{\varphi_{\star}}(x, s, \cdot)$, so that by Levi's monotone convergence theorem,

$$
\int_{\Omega_{\ell}} \overline{\varphi_{\star}}\left(x, u^{\star}, \nabla u^{\star}\right) \leqslant \int_{\Omega_{\ell}} \bar{\varphi}(x, u, \nabla u) .
$$

The conclusion comes from Levi's monotone convergence theorem for $\ell \rightarrow \infty$ and from the fact that $\bigcup_{\ell \in \mathbb{N}} \Omega_{\ell}=\Omega$ up to a set of zero measure. 
Proposition 5.18. Let $I: \Omega \times \mathbb{R}^{+} \times \mathbb{R}^{N} \rightarrow \overline{\mathbb{R}}^{+}$be lower semi-continuous, suppose $I(\cdot, s, \xi)$ is totally invariant with respect to $L$ and, for any $x \in \Omega, I(x, \cdot)$ is convex and $\lim _{|s|+|\xi|_{2} \rightarrow \infty} I(x, s, \xi)=+\infty$. If

$$
\int_{\Omega} I(x, u, \nabla u) \mathrm{d} x<\infty,
$$

then for any $x \in \Omega$, there exists a neighborhood $\mathcal{N}_{x}$ totally invariant with respect to $L$ such that for any $M>0$,

$$
\sup _{\substack{A \subset \mathcal{N}_{x} \\ \mathcal{L}^{N}(A)=M}} \int_{A}|\nabla u|_{2}+|u| \mathrm{d} x<+\infty .
$$

Proof. Let $x \in \mathbb{R}^{N}$. Since $I(x, \cdot)$ is coercive, there exists $R>0$ such that $I(x, s, \xi)>1$ if $|s|+|\xi|_{2}=R$. Since $I$ is lower semi-continuous and the set $\{x\} \times\left\{(s, \xi):|s|+|\xi|_{2}=R\right\}$ is compact, this remains true in a neighborhood of this set. Hence, there is a neighborhood $\mathcal{N}_{x}$ of $x$ such that $I(y, s, \xi)>1$ if $|s|+|\xi|_{2}=R$ and $y \in \mathcal{N}_{x}$. Since $I(\cdot, s, \xi)$ is totally invariant, without loss of generality, $\mathcal{N}_{x}$ is totally invariant. By convexity of $I(x, \cdot), I(y, s, \xi)>\left(|s|+\left.|\xi|\right|_{2}\right) / R$ if $\left(|s|+|\xi|_{2}\right) \geqslant R$. Therefore $I(y, s, \xi)>\left(|s|+|\xi|_{2}\right) / R-1$. If $A \subset \mathcal{N}_{x}$ and $\mathcal{L}^{N}(A)=M$, then

$$
\begin{aligned}
\int_{A}|(u, \nabla u)|_{2} \mathrm{~d} x & \leqslant R \int_{A} I(x, u, \nabla u)+1 \mathrm{~d} x \leqslant R \int_{A} I(x, u, \nabla u) \mathrm{d} x+R \mathcal{L}^{N}(A) \\
& \leqslant R \int_{\Omega} I(x, u, \nabla u)+R M<+\infty .
\end{aligned}
$$

Since the right-hand side does not depend on $A$, the proof is complete.

\section{Inequalities for anisotropic symmetrization}

Definition 6.1. The vector $t \in \mathbb{R}^{N}$ is a subdifferential of $f: \mathbb{R}^{N} \rightarrow \overline{\mathbb{R}}$ at $x \in \mathbb{R}^{N}$ if for all $y \in \mathbb{R}^{N}$,

$$
f(y) \geqslant f(x)+\langle t, y-x\rangle .
$$

The set of the subdifferentials of $f$ at $x$ is denoted $\partial f(x)$.

It is standard in the theory of convex functions that if $\varphi: \mathbb{R}^{N} \rightarrow \mathbb{R}$ is convex, then $\partial \varphi(x)$ is nonempty for every $x \in \mathbb{R}^{N}$. If $\varphi$ is also Gateaux-differentiable at $x \in \mathbb{R}^{N}$, then $\partial \varphi(x)=\{\nabla \varphi(x)\}$.

Proposition 6.2. If $H \in \mathcal{H}\left(\mathbb{R}^{k}\right)$, then for all $t \in \mathbb{R}^{k}$, there exists $x \in \partial H^{\circ}(t)$ such that $H(x)=1$. In particular, if $H^{\circ}$ is differentiable at $t, H\left(\nabla H^{\circ}(t)\right)=1$.

Proof. Let $t \in \mathbb{R}^{k}$. By definition of $H^{\circ}$ and by positive homogeneity of $H$,

$$
H^{\circ}(t)=\sup _{\substack{y \in \mathbb{R}^{N} \\ y \neq 0}} \frac{\langle t, y\rangle}{H(y)}=\sup _{|y|_{2}=1} \frac{\langle t, y\rangle}{H(y)} .
$$

Since the function on right-hand side is upper semi-continuous, its least upper bound is attained for some $x \in \mathbb{R}^{N}$. Since $H$ is positively homogeneous, without loss of generality, $H(x)=1$ and $H^{\circ}(t)=\langle t, x\rangle$. For any $s \in \mathbb{R}^{k}$

$$
H^{\circ}(s) \geqslant\langle s, x\rangle=H^{\circ}(t)+\langle s-t, x\rangle .
$$

Lemma 6.3. If $G_{1}, G_{2}: \mathbb{R}^{N} \rightarrow \mathbb{R}^{+}$are gauges, then the function

$$
\Psi: \mathbb{R}^{N} \rightarrow \mathbb{R}^{N}: x \mapsto x \frac{G_{1}(x)}{G_{2}(x)}
$$

is Lipschitz-continuous. 
Proof. Since $\left|G_{i}(x)-G_{i}(y)\right| \leqslant G_{i}(x-y)$, there holds

$$
\begin{aligned}
& |\Psi(x)-\Psi(y)|_{2} \\
& \quad \leqslant\left|x \frac{G_{1}(x)}{G_{2}(x)}-x \frac{G_{1}(y)}{G_{2}(x)}\right|_{2}+\left|x \frac{G_{1}(y)}{G_{2}(x)}-x \frac{G_{1}(y)}{G_{2}(y)}\right|_{2}+\left|x \frac{G_{1}(y)}{G_{2}(y)}-y \frac{G_{1}(y)}{G_{2}(y)}\right|_{2} \\
& \quad \leqslant|x-y|_{2}\left[\sup _{z \in \mathbb{R}^{N}} \frac{G_{1}(z)}{|z|_{2}} \cdot \sup _{x \in \mathbb{R}^{N}} \frac{|x|_{2}}{G_{2}(x)}+\sup _{x \in \mathbb{R}^{N}} \frac{|x|_{2}}{G_{2}(x)} \cdot \sup _{y \in \mathbb{R}^{N}} \frac{G_{1}(y)}{G_{2}(y)} \cdot \sup _{z \in \mathbb{R}^{N}} \frac{G_{2}(z)}{|z|_{2}}+\sup _{z \in \mathbb{R}^{N}} \frac{G_{1}(z)}{G_{2}(z)}\right] .
\end{aligned}
$$

Since all least upper bounds can be restricted to the unit sphere in $\mathbb{R}^{N}$ and $G_{1}, G_{2}$ and $|\cdot|_{2}$ are continuous and do not vanish on the unit sphere, the function $\Psi$ is Lipschitz-continuous.

Proposition 6.4 ((Gauge change of variable)). Let $\Omega^{\prime \prime} \subset \mathbb{R}^{N-k}, \Omega=\mathbb{R}^{k} \times \Omega^{\prime \prime}, w: \mathbb{R}^{+} \times \Omega^{\prime \prime} \rightarrow \mathbb{R}, H \in \mathcal{H}\left(\mathbb{R}^{k}\right)$, $u(x)=w\left(K_{H^{\circ}}^{-1}\left|x^{\prime}\right|_{2}, x^{\prime \prime}\right)$ and $v(x)=\left(H^{\circ}\left(-x^{\prime}\right), x^{\prime \prime}\right)$. Then, $u \in W_{\mathrm{loc}}^{1,1}(\Omega)$ if and only if $v \in W_{\mathrm{loc}}^{1,1}(\Omega)$.

Furthermore, for any $f: \Omega^{\prime \prime} \times \mathbb{R}^{+} \times \mathbb{R}^{+} \times \mathbb{R}^{N-k} \rightarrow \mathbb{R}$,

$$
\int_{\Omega} f\left(x^{\prime \prime}, v, H\left(\nabla^{\prime} v\right), \nabla^{\prime \prime} v\right) \mathrm{d} x=\int_{\mathbb{R}^{N}} f\left(x^{\prime \prime}, u, K_{H^{\circ}}\left|\nabla^{\prime} u\right|_{2}, \nabla^{\prime \prime} u\right) \mathrm{d} x,
$$

provided one of the integrals exists.

Remark 6.5. Recall that for any gauge $G: \mathbb{R}^{k} \rightarrow \mathbb{R}, K_{G}$ is the positive constant given by Definition 2.4.

Proof. Since $v$ is a obtained by a bi-Lipschitzian mapping from weakly differentiable $u$, it is also weakly differentiable [31, Theorem 2.2.2, p. 52].

Since $H^{\circ}$ is Lipschitz, it is almost everywhere differentiable, its weak derivative coincides almost everywhere with its unique subgradient, and, by Proposition 6.2, $H\left(-\nabla H^{\circ}\left(-x^{\prime}\right)\right)=1$ almost everywhere. Hence

$$
\begin{aligned}
& f\left(x^{\prime \prime}, u, K_{H^{\circ}}\left|\nabla^{\prime} u\right|_{2}, \nabla^{\prime \prime} u\right)\left(x^{\prime}, x^{\prime \prime}\right)=f\left(x^{\prime \prime}, w,-\partial_{r} w, \nabla^{\prime \prime} w\right)\left(K_{H^{\circ}}^{-1}\left|x^{\prime}\right|_{2}, x^{\prime \prime}\right), \\
& \begin{aligned}
f\left(x^{\prime \prime}, v, H\left(\nabla^{\prime} v\right), \nabla^{\prime \prime} v\right)\left(x^{\prime}, x^{\prime \prime}\right) & =f\left(x^{\prime \prime}, w\left(H^{\circ}\left(-x^{\prime}\right), x^{\prime \prime}\right), H\left(\partial_{r} w, \nabla H^{\circ}\left(-x^{\prime}\right)\right), \nabla^{\prime \prime} w\left(H^{\circ}\left(-x^{\prime}\right), x^{\prime \prime}\right)\right) \\
= & f\left(x^{\prime \prime}, w,-\partial_{r} w, \nabla^{\prime \prime} w\right)\left(H^{\circ}\left(-x^{\prime}\right), x^{\prime \prime}\right) .
\end{aligned}
\end{aligned}
$$

Since $\mathcal{L}^{k}\left(\left\{H^{\circ}\left(-x^{\prime}\right)<\lambda\right\}\right)=\mathcal{L}^{k}\left(\left\{\left|x^{\prime}\right|_{2}<\lambda K_{H^{\circ}}\right\}\right)$ for all $\lambda \in \mathbb{R}$, the equality follows.

Corollary 6.6. Let $H \in \mathcal{H}\left(\mathbb{R}^{k}\right), \cdot^{*}$ denote the $(G, L, T)$-anisotropic symmetrization and ${ }^{\star}$ denote the Steiner symmetrization with respect to $T$. If $\Omega$ is totally invariant with respect to $.^{*}, f: \mathbb{R}^{+} \times \mathbb{R}^{N} \times \Omega \rightarrow \mathbb{R}, f(\cdot, s, \eta, \xi)$ is totally invariant for each $(s, \eta, \xi) \in \mathbb{R}^{+} \times \mathbb{R}^{+} \times \mathbb{R}^{N-k}$, u vanishes at the infinity with respect to ${ }^{*}$ and $u^{*} \in W_{\mathrm{loc}}^{1,1}(\Omega)$, then

$$
\int_{\mathbb{R}^{N}} f\left(x, u^{*}, H\left(\nabla^{\prime} u^{*}\right), \nabla^{\prime \prime} u^{*}\right) \mathrm{d} x=\int_{\mathbb{R}^{N}} f\left(u^{\star}, K_{H^{\circ}}\left|\nabla^{\prime} u^{\star}\right|_{2}, \nabla^{\prime \prime} u^{\star}\right) \mathrm{d} x,
$$

provided one of the integrals exist.

Lemma 6.7. Let $J: \mathbb{R}^{+} \times \mathbb{R}^{N-k} \rightarrow \overline{\mathbb{R}}^{+}, H \in \mathcal{H}\left(\mathbb{R}^{k}\right)$, and

$$
\varphi(x)=J\left(H^{\circ}\left(x^{\prime}\right), x^{\prime \prime}\right) .
$$

If $J(0)=0$, then

$$
\bar{\varphi}(t)=\bar{J}\left(H^{\circ \circ}\left(t^{\prime}\right), t^{\prime \prime}\right) \leqslant \bar{J}\left(H\left(t^{\prime}\right), t^{\prime \prime}\right) .
$$

Proof. By definition of the Fenchel transform, 


$$
\begin{aligned}
\bar{\varphi}(t) & =\sup _{x \in \mathbb{R}^{N}}\langle t, x\rangle-J\left(H^{\circ}\left(x^{\prime}\right), x^{\prime \prime}\right)=\sup _{\substack{\lambda \in \mathbb{R}^{+} \\
x \in \mathbb{R}^{N}, H^{\circ}\left(x^{\prime}\right)=\lambda}}\left\langle t^{\prime}, x^{\prime}\right\rangle+\left\langle t^{\prime \prime}, x^{\prime \prime}\right\rangle-J\left(\lambda, x^{\prime \prime}\right) \\
= & \sup _{\substack{\lambda \in \mathbb{R}^{+} \\
x^{\prime \prime} \in \mathbb{R}^{N-k}}} H^{\circ \circ}\left(t^{\prime}\right) \lambda+\left\langle t^{\prime \prime}, x^{\prime \prime}\right\rangle-J\left(\lambda, x^{\prime \prime}\right)=\bar{J}\left(H^{\circ \circ}\left(t^{\prime}\right), t^{\prime \prime}\right) \leqslant \bar{J}\left(H\left(t^{\prime}\right), t^{\prime \prime}\right),
\end{aligned}
$$

the last inequality coming from the convexity of $\bar{J}$, and the fact $\bar{J}(0)=0$ and $\bar{J} \geqslant 0$.

Theorem 6.8. Let $H \in \mathcal{H}\left(\mathbb{R}^{k}\right)$, .* be the anisotropic symmetrization with respect to $H^{\circ}$, let $\Omega \subset \mathbb{R}^{N}$ be an open set totally invariant with respect to ${ }^{*}$ and $J: \Omega \times \mathbb{R}^{+} \times \mathbb{R}^{+} \times \mathbb{R}^{N-k} \rightarrow \overline{\mathbb{R}}^{+}$. If $J(\cdot, s, \eta, \xi)$ is totally invariant for each $(s, \eta, \xi) \in \mathbb{R}^{+} \times \mathbb{R}^{+} \times \mathbb{R}^{N-k}, J(x, s, \cdot, \cdot)$ is convex and lower semi-continuous for each $(x, s) \in \Omega \times \mathbb{R}^{+}, \bar{J}(\cdot, \cdot, \eta, \xi)$ is lower semi-continuous for each $(\eta, \xi) \in \mathbb{R}^{+} \times \mathbb{R}^{N-k}$ and $u \in W_{\text {loc },+}^{1,1}(\Omega)$, and there exists $I: \Omega \times \mathbb{R}^{+} \times \mathbb{R}^{N} \rightarrow \mathbb{R}^{+}$ such that $I$ is lower semi-continuous, $I(\cdot, s, \xi)$ is totally invariant for each $(s, \xi) \in \mathbb{R}^{+} \times \mathbb{R}^{N}, I(x, \cdot)$ is convex and $\lim _{|s|+|\xi|_{2} \rightarrow \infty} I(x, s, \xi)=+\infty$ for each $x \in \Omega$ and

$$
\int_{\Omega} I(x, u, \nabla u) \mathrm{d} x<\infty,
$$

then $u^{*} \in W_{\mathrm{loc}}^{1,1}(\Omega)$ and

$$
\int_{\Omega} J\left(x, u^{*}, H\left(\nabla^{\prime} u^{*}\right), \nabla^{\prime \prime} u^{*}\right) \mathrm{d} x \leqslant \int_{\Omega} J\left(x, u, H\left(\nabla^{\prime} u\right), \nabla^{\prime \prime} u\right) \mathrm{d} x .
$$

Proof. Let $\varphi(x, s, t)=\bar{J}\left(x, s, H^{\circ}\left(t^{\prime}\right), t^{\prime \prime}\right)$. Then, by Lemma 6.7,

$$
\begin{aligned}
& \bar{\varphi}(x, s, \theta)=J\left(x, s, H^{\circ \circ}\left(\theta^{\prime}\right), \theta^{\prime \prime}\right) \leqslant J\left(x, s, H\left(\theta^{\prime}\right), \theta^{\prime \prime}\right), \\
& \varphi^{\star}(x, s, t)=\bar{J}\left(x, s,\left|t^{\prime}\right|_{2} / K_{H^{\circ}}, t^{\prime \prime}\right), \\
& \overline{\varphi^{\star}}(x, s, \theta)=J\left(x, s, K_{\left.H^{\circ}\left|\theta^{\prime}\right|_{2}, \theta^{\prime \prime}\right) .}\right.
\end{aligned}
$$

The function $u$ verifies the hypotheses of Proposition 5.17 by Proposition 5.18. Hence, by Proposition 5.17

$$
\int_{\Omega} J\left(x, u^{\star}, K_{H^{\circ}}\left|\nabla^{\prime} u^{\star}\right|_{2}, \nabla^{\prime \prime} u^{\star}\right) \mathrm{d} x \leqslant \int_{\Omega} J\left(x, u, H\left(\nabla^{\prime} u\right), \nabla^{\prime \prime} u\right) \mathrm{d} x .
$$

The conclusion comes from Proposition 6.4.

Theorem 6.9 ((Anisotropic symmetrization inequality for anisotropic functional)). Let $*^{*}$ be the anisotropic symmetrization with respect to a gauge $G: \mathbb{R}^{k} \rightarrow \mathbb{R}^{+}$, let $\Omega$ be a totally invariant open set, $\varphi: \Omega \times \mathbb{R}^{+} \times \mathbb{R}^{N} \rightarrow \overline{\mathbb{R}}^{+}$ and $u \in W_{\text {loc, }+}^{1,1}(\Omega)$. If $\varphi(\cdot, \cdot, 0)=0, \varphi(\cdot, s, \xi)$ is totally invariant with respect to ${ }^{\star}$ for each $(s, \xi) \in \mathbb{R}^{+} \times \mathbb{R}^{N}$ and $\varphi(\cdot, \cdot, \xi)$ is lower semi-continuous for each $\xi \in \mathbb{R}^{N}$, and there exists $I: \Omega \times \mathbb{R}^{+} \times \mathbb{R}^{N} \rightarrow \overline{\mathbb{R}}^{+}$such that I is lower semi-continuous, $I(\cdot, s, \xi)$ is totally invariant for each $(s, \xi) \in \mathbb{R}^{+} \times \mathbb{R}^{+} \times \mathbb{R}^{N-k}, I(x, \cdot)$ is convex and $\lim _{|s|+|\xi|_{2} \rightarrow \infty} I(x, s, \xi)=+\infty$ for each $x \in \Omega$, and

$$
\int_{\Omega} I(x, u, \nabla u) \mathrm{d} x<\infty,
$$

then $u^{*} \in W_{\text {loc }}^{1,1}(\Omega)$ and

$$
\int_{\Omega} \overline{\varphi_{*}}\left(x, u^{*}(x), \nabla u^{*}(x)\right) \mathrm{d} x \leqslant \int_{\Omega} \bar{\varphi}(x, u(x), \nabla u(x)) \mathrm{d} x,
$$

where the symmetrization and the Fenchel transform of $\varphi$ are taken with respect to the last variable. 
Proof. There exists a unique function $J: \Omega \times \mathbb{R}^{+} \times \mathbb{R}^{+} \times \mathbb{R}^{N-k} \rightarrow \overline{\mathbb{R}}^{+}$such that for each $(x, s, \xi) \in \Omega \times \mathbb{R}^{+} \times \mathbb{R}^{N}$,

$$
\begin{aligned}
& \varphi_{\star}\left(x, s, \xi^{\prime}, \xi^{\prime \prime}\right)=J\left(x, s, K_{G}^{-1}\left|\xi^{\prime}\right|_{2}, \xi^{\prime \prime}\right), \\
& \varphi_{*}\left(x, s, \xi^{\prime}, \xi^{\prime \prime}\right)=J\left(x, s, G\left(-\xi^{\prime}\right), \xi^{\prime \prime}\right) ;
\end{aligned}
$$

Lemma 6.7 implies

$$
\begin{aligned}
& \overline{\varphi_{\star}}\left(x, s, t^{\prime}, t^{\prime \prime}\right)=\bar{J}\left(x, s, K_{G}\left|t^{\prime}\right|_{2}, t^{\prime \prime}\right), \\
& \overline{\varphi_{*}}\left(x, s, t^{\prime}, t^{\prime \prime}\right)=\bar{J}\left(x, s, G^{\circ}\left(-t^{\prime}\right), t^{\prime \prime}\right) ;
\end{aligned}
$$

with Proposition 6.4 we have

$$
\int_{\Omega} \overline{\varphi_{*}}\left(x, s, \nabla u^{*}\right) \mathrm{d} x=\int_{\Omega} \overline{\varphi_{\star}}\left(x, s, \nabla u^{\star}\right) \mathrm{d} x ;
$$

and the conclusion follows from Proposition 5.6.

Definition 6.10. For $u \in W_{\text {loc },+}^{1,1}(\Omega), F \in \mathcal{H}\left(\mathbb{R}^{k}\right)$ and a Borel measurable function $J: \Omega \times \mathbb{R}^{+} \times \mathbb{R}^{+} \times \mathbb{R}^{N-k} \rightarrow \mathbb{R}^{+}$ such that $J(x, \cdot)$ is convex for each $x \in \Omega$, let

$$
\|u\|_{J, F, \Omega}=\inf \left\{\lambda>0: \int_{\Omega} J\left(x, \frac{u}{\lambda}, F\left(\frac{\nabla^{\prime} u}{\lambda}\right), \frac{\nabla^{\prime \prime} u}{\lambda}\right) \mathrm{d} x<+\infty\right\}
$$

and let

$$
W_{+}^{1, J, F}(\Omega)=\left\{u \in W_{\mathrm{loc},+}^{1,1}(\Omega):\|u\|_{J, F, \Omega}<+\infty\right\} .
$$

Corollary 6.11. Suppose $F: \mathbb{R}^{k} \rightarrow \mathbb{R}$ is a gauge, $\cdot^{*}$ is the anisotropic symmetrization with respect to $F^{\circ}, \Omega \subset \mathbb{R}^{N}$ is a totally invariant open set and $J: \Omega \times \mathbb{R}^{+} \times \mathbb{R}^{+} \times \mathbb{R}^{N-k} \rightarrow \mathbb{R}^{+}$is lower semi-continuous, $J(x, s, 0,0)=0$ for each $(x, s) \in \Omega \times \mathbb{R}^{+}, J(x, \cdot)$ is convex for each $x \in \Omega, \bar{J}(\cdot, \cdot, \eta, \xi)$ is lower semi-continuous for each $(\eta, \xi) \in \mathbb{R}^{+} \times \mathbb{R}^{N-k}$, and for each $x \in \Omega$,

$$
\lim _{|s|+|\eta|+|\xi|_{2} \rightarrow \infty} J(x, s, \eta, \xi)=\infty .
$$

If $u \in W_{+}^{1, J, F}(\Omega)$ vanishes at the infinity with respect to $\cdot^{*}$, then $u^{*} \in W_{+}^{1, J, F}(\Omega)$ and

$$
\left\|u^{*}\right\|_{J, F, \Omega} \leqslant\|u\|_{J, F, \Omega} .
$$

\section{Applications}

\subsection{Anisotropic isoperimetric inequalities}

The results can be extended to $B V\left(\mathbb{R}^{N}\right)$ and to isoperimetric inequalities. Since our approach uses perimeters in the sense of Cacciopoli defined by duality, it cannot prove anything for non-convex perimeter functions like the ones arising in Wulff's theory of crystals.

Definition 7.1. For any $u \in L^{1}\left(\mathbb{R}^{N}\right)$, let

$$
P_{H}(u)=\sup \left\{\sum_{i=1}^{N} \int u \frac{\partial h_{i}}{\partial x_{i}}: h \in \mathcal{D}\left(\mathbb{R}^{N}\right), \forall x \in \mathbb{R}^{N}, H^{\circ}(-h(x)) \leqslant 1\right\} .
$$

Theorem 7.2 ((Anisotropic isoperimetric inequality in $\left.\left.B V\left(\mathbb{R}^{N}\right)\right)\right)$. Let $H: \mathbb{R}^{N} \rightarrow \mathbb{R}$ be a gauge. Let.$^{*}$ denote the anisotropic symmetrization with respect to $H^{\circ}$. If $u \in L^{1}\left(\mathbb{R}^{N}\right)$, then

$$
P_{H}\left(u^{*}\right) \leqslant P_{H}(u)
$$


Proof. This will be deduced as a corollary of Theorem 6.8. Note that $P_{H}$ is convex and lower semi-continuous in $L^{1}\left(\mathbb{R}^{N}\right)$. If $u \in \mathcal{D}\left(\mathbb{R}^{N}\right)$, then

$$
P_{H}(u)=\sup \left\{\int_{\mathbb{R}^{N}}\langle\nabla u, h\rangle: H^{\circ}(h) \leqslant 1\right\}=\int_{\mathbb{R}^{N}} H^{\circ}(\nabla u) .
$$

Let $\left(\rho_{n}\right)_{n \in \mathbb{N}}$ be a sequence of nonnegative smooth mollifiers. By lower semi-continuity of $P_{H}$,

$$
\liminf _{n \rightarrow \infty} P_{H}\left(\rho_{n} * u\right) \geqslant P_{H}(u) \text {. }
$$

Conversely, for $h \in \mathcal{D}\left(\mathbb{R}^{N}\right)$, if $H(-h) \leqslant 1$, then the convexity of $H$ implies $H\left(-\rho_{n} * h\right) \leqslant 1$, whence

$$
P_{H}\left(\rho_{n} * u\right) \leqslant P_{H}(u) \text {. }
$$

We have thus $\lim _{n \rightarrow \infty} P_{H}\left(\rho_{n} * u\right)=P_{H}(u)$. Since $\rho_{n} * u \rightarrow u$ in $L^{1}\left(\mathbb{R}^{N}\right)$, then $\left(\rho_{n} * u\right)^{*} \rightarrow u^{*}$ in $L^{1}\left(\mathbb{R}^{N}\right)$. Since $P_{H}$ is lower semi-continuous in $L^{1}\left(\mathbb{R}^{N}\right)$, by Theorem 6.8 ,

$$
P_{H}\left(u^{*}\right) \leqslant \liminf _{n \rightarrow \infty} P_{H}\left(\rho_{n} * u^{*}\right) \leqslant \liminf _{n \rightarrow \infty} P_{H}\left(\rho_{n} * u\right)=P_{H}(u)
$$

A consequence of this proposition is the following isoperimetric inequality: For any measurable set $A \subset \mathbb{R}^{N}$,

$$
P_{H}\left(A^{*}\right)=P_{H}\left(\chi_{A^{*}}\right) \leqslant P_{H}(A)=P_{H}\left(\chi_{A}\right) .
$$

\subsection{Anisotropic Sobolev and Hardy-Sobolev inequalities}

Proposition 7.3. Suppose $F \in \mathcal{H}\left(\mathbb{R}^{k}\right), \cdot^{*}$ is the anisotropic symmetrization with respect to $F^{\circ}, \Omega \subset \mathbb{R}^{N}$ is a totally invariant open set and $J: \Omega \times \mathbb{R}^{+} \times \mathbb{R}^{+} \times \mathbb{R}^{N-k} \rightarrow \mathbb{R}^{+}$is lower semi-continuous, $J(x, \cdot)$ is convex for each $x \in \Omega$, $J(x, s, 0,0)=0$ for each $(x, s) \in \Omega \times \mathbb{R}^{+}, \bar{J}(\cdot, \cdot, \eta, \xi)$ is lower semi-continuous for each $(\eta, \xi) \in \mathbb{R}^{+} \times \mathbb{R}^{N-k}$, and for each $x \in \Omega, \lim _{|s|+|\eta|+|\xi|_{2} \rightarrow \infty} J(x, s, \eta, \xi)=\infty$. Suppose $\|\cdot\|_{X}$ is a norm that is invariant with respect to $\cdot^{*}$. Suppose for any $u \in W_{+}^{1, J, F}(\Omega)$ that vanishes at the infinity with respect to $\cdot^{*}$,

$$
\|u\|_{X} \leqslant\|u\|_{J, F, \Omega}
$$

then for any $E \in \mathcal{H}\left(\mathbb{R}^{k}\right)$ such that $K_{E^{\circ}}=K_{F^{\circ}}$,

$$
\|u\|_{X} \leqslant\|u\|_{J, E, \Omega} .
$$

If there exists $u \in W_{+}^{1, J, F}(\Omega)$ that vanishes at the infinity with respect to $\cdot^{*}$ such that $\|u\|_{X}=\|u\|_{J, F, \Omega}$, then $\left\|u^{\star}\right\|_{X}=$ $\left\|u^{\star}\right\|_{J, E, \Omega}$, where $\cdot^{\star}$ denotes the anisotropic symmetrization with respect to $E^{\circ}$.

Proof. First note that $\|\cdot\|_{X}$ is invariant with respect to ${ }^{\star}$. In fact, for any $u,\|u\|_{X}=\left\|u^{*}\right\|_{X}=\left\|u^{\star}\right\|_{X}$, since $u^{\star *}=u^{*}$ and $\|\cdot\|_{X}$ is invariant with respect to $*^{*}$.

It is then clear that

$$
\|u\|_{X}=\left\|u^{*}\right\|_{X} \leqslant\left\|u^{*}\right\|_{F, J, \Omega}=\left\|u^{\star}\right\|_{E, J, \Omega} \leqslant\|u\|_{E, J, \Omega},
$$

where the first inequality comes from the hypothesis (7.1) and the second from Corollary 6.11. The conclusions follow.

Remark 7.4. Sobolev inequalities thus do not rely essentially neither on the convexity nor on the evenness of the Euclidian norm. It is not surprising that such inequalities are possible since for any $F \in \mathcal{H}\left(\mathbb{R}^{k}\right)$, there exists $\alpha>0$ such that $F(y) \geqslant \alpha|y|_{2}$. The striking fact is that optimal Sobolev-Orlicz constants depend on $F \in \mathcal{H}\left(\mathbb{R}^{F}\right)$ only through $K_{H^{\circ}}$.

Proposition 7.5. For any $H \in \mathcal{H}\left(\mathbb{R}^{N}\right)$ and any $u \in \mathcal{D}\left(\mathbb{R}^{N}\right)$ and $1<p<N$,

$$
\int_{\mathbb{R}^{N}} H(\nabla u)^{p} \mathrm{~d} x \geqslant\left(\frac{N-p}{p}\right)^{p} \int_{\mathbb{R}^{N}} \frac{|u|^{p}}{H^{\circ}(x)^{p}} \mathrm{~d} x .
$$

The constant is optimal for any fixed $H \in \mathcal{H}\left(\mathbb{R}^{k}\right)$. 
Proof. Without loss of generality, $K_{H^{\circ}}=1$. Let $*^{\star}$ denote the Schwarz symmetrization. Then, by Theorem 6.8 , the classical Hardy-Sobolev inequality [30] and by Proposition 2.28,

$$
\int_{\mathbb{R}^{N}} H(\nabla u)^{p} \mathrm{~d} x \geqslant \int_{\mathbb{R}^{N}}\left|\nabla u^{\star}\right|_{2}^{p} \mathrm{~d} x \geqslant\left(\frac{N-p}{p}\right)^{p} \int_{\mathbb{R}^{N}} \frac{\left|u^{\star}\right|^{p}}{|x|_{2}^{p}} \mathrm{~d} x \geqslant\left(\frac{N-p}{p}\right)^{p} \int_{\mathbb{R}^{N}} \frac{|u|^{p}}{H^{\circ}(x)^{p}} \mathrm{~d} x .
$$

The fact that the constant is optimal comes from the same reasoning with the symmetrization ** with respect to $H^{\circ}$ and the fact that the constant is optimal in the isotropic case [30].

\subsection{Recovering continuity and compactness}

Proposition 7.6. Suppose $H \in \mathcal{H}\left(\mathbb{R}^{k}\right), J: \mathbb{R}^{+} \times \mathbb{R}^{N-k} \rightarrow \mathbb{R}^{+}, J(t, \cdot)$ is a gauge for each $t \in \mathbb{R}^{+}$and $1<p<N$. Let $*^{*}$ denote the anisotropic symmetrization with respect to $H^{\circ}$ and $\Omega$ be totally invariant with respect to $\cdot^{*}$. If $f \in L^{q}(\Omega), q^{-1}+p^{-1}=1+N^{-1}$ and $f^{*}=f$, then the function $E: \mathcal{D}_{0,+}^{1, p}(\Omega) \rightarrow \mathbb{R}$ (where $\mathcal{D}_{0}^{1, p}$ is the completion of $\mathcal{D}(\Omega)$ with respect to the norm $\left.\|u\|=\left(\int_{\Omega}|\nabla u|^{p}\right)^{1 / p}\right)$,

$$
u \mapsto E(u)=\int_{\mathbb{R}^{N}} J\left(H\left(\nabla^{\prime} u\right), \nabla^{\prime \prime} u\right)^{p}-f u \mathrm{~d} x
$$

has a minimizer $u=u^{*}$.

Remark 7.7. If $\left(u_{n}\right)$ is a minimizing sequence for $E$, it is bounded in $\mathcal{D}^{1, p}(\Omega)$, hence up to a subsequence, $u_{n} \rightarrow u$ in $\mathcal{D}^{1, p}(\Omega)$. Then

$$
\int_{\Omega} f u_{n} \mathrm{~d} x \rightarrow \int_{\Omega} f u \mathrm{~d} x .
$$

But if $H$ is not convex it is not true in general that

$$
\int_{\Omega} J\left(H\left(\nabla^{\prime} u\right), \nabla^{\prime \prime} u\right)^{p} \mathrm{~d} x \leqslant \liminf _{n \rightarrow \infty} \int_{\Omega} J\left(H\left(\nabla^{\prime} u_{n}\right), \nabla^{\prime \prime} u_{n}\right)^{p} \mathrm{~d} x
$$

(see [11]).

In a different setting, the symmetry of the domain helps to recover the existence for some nonconvex problems [16]. On the other hand, some crystalline problems close to Wulff's problem do not have any solution (except in the varifold sense) when the energy is not convex [27].

Remark 7.8. Even if the minimizer is unique (if e.g. $J$ is strictly convex), that does not guarantee the symmetry of the minimizer since, except in the radial case, the problem is not invariant under the action of a continuous group.

Proof. Let $u_{n}$ be a minimizing sequence. Then $\left(v_{n}\right)=\left(u_{n}^{*}\right)$ is also a minimizing sequence. Up to a subsequence $v_{n} \rightarrow v$ in $\mathcal{D}_{0}^{1, p}\left(\mathbb{R}^{N}\right)$, hence

$$
\int_{\Omega} f v_{n} \mathrm{~d} x \rightarrow \int_{\Omega} f v \mathrm{~d} x
$$

Furthermore

$$
\int_{\Omega} J\left(H\left(\nabla^{\prime} v\right), \nabla^{\prime \prime} v\right)^{p} \mathrm{~d} x \leqslant \liminf _{n \rightarrow \infty} \int_{\Omega} J\left(H\left(\nabla^{\prime} v_{n}\right), \nabla^{\prime \prime} v_{n}\right)^{p} \mathrm{~d} x,
$$

since the functional $u \mapsto \int_{\Omega} J\left(H\left(\nabla^{\prime} u\right), \nabla^{\prime \prime} u\right)^{p} \mathrm{~d} x$ is convex on the image set of .*. Then $v$ is a minimizer for the functional $E$. Hence $v=v^{*}$ is a minimizer of $E$. 
Proposition 7.9. Suppose $H \in \mathcal{H}\left(\mathbb{R}^{k}\right), \cdot^{*}$ is the anisotropic symmetrization with respect to $H^{\circ}, \Omega$ is totally invariant with respect to $\cdot^{*}, J: \Omega \times \mathbb{R}^{+} \times \mathbb{R}^{+} \times \mathbb{R}^{N-k} \rightarrow \mathbb{R}^{+}$is such that $\bar{J}(\cdot, \cdot, \eta, \xi)$ is lower semi-continuous and totally invariant with respect to ${ }^{*}$ for each $(\eta, \xi) \in \mathbb{R}^{+} \times \mathbb{R}^{N-k}$, and $J(x, \cdot)$ is convex for each $x \in \Omega$. Let $f: \Omega \times \mathbb{R}^{+} \rightarrow \mathbb{R}^{+}$ be such that for almost every $x \in \Omega, f(x, 0)=0$ and $f(x, \cdot)$ is continuous for each $x \in \Omega$ and $f(\cdot, s)$ is measurable and totally invariant for each $s \in \mathbb{R}^{+}$.

For any $u \in W_{0,+}^{1, p}(\Omega)$, let

$$
E(u)=\int_{\Omega} J\left(x, u(x), H\left(\nabla^{\prime} u(x)\right), \nabla^{\prime \prime} u(x)\right) \mathrm{d} x,
$$

and

$$
\mathcal{M}=\left\{u \in W_{0,+}^{1, p}(\Omega): \int_{\Omega} f(x, u(x)) \mathrm{d} x=1\right\} .
$$

If there exists $1<p<+\infty, 0<\alpha<\beta, p<q<\frac{N p}{N-p}$ and $\gamma>0$ such that

$$
\alpha\left(|s|+|\eta|+|\xi|_{2}\right)^{p} \leqslant J(x, s, \eta, \xi) \leqslant \beta\left(|s|+|\eta|+|\xi|_{2}\right)^{p}
$$

and

$$
|f(x, s)| \leqslant \gamma\left(|s|^{p}+|s|^{q}\right)
$$

and if $\lim _{s \rightarrow 0} \sup _{x \in \Omega}(|f(x, s)|) /|s|^{p}=0$, then there exists $u \in \mathcal{M}$ such that $E(u)=\inf _{v \in \mathcal{M}} E(v)$. Furthermore, $u^{*}=u$.

Proof. Let $\left(u_{n}\right)_{n \in \mathbb{N}}$ be a minimizing sequence of $E$ in $\mathcal{M}$. By Theorem 6.8 with $I(x, s, \xi)=\alpha\left(|s|+\left|\xi^{\prime}\right|_{2}+\left|\xi^{\prime \prime}\right|_{2}\right)^{p}$, $E\left(u_{n}^{*}\right) \leqslant E\left(u_{n}\right)$ and by Proposition 2.28, $u_{n}^{*} \in \mathcal{M}$. Without loss of generality, $u_{n}^{*}=u_{n}$ for each $n \in \mathbb{N}$. Since $E\left(u_{n}\right)$ is bounded, the sequence $\left(u_{n}\right)$ is bounded in $W_{0}^{1, p}(\Omega)$. Hence up to a subsequence it converges weakly to $u \in W_{0}^{1, p}(\Omega)$. By Lemma 3.1, $u^{*}=u$. Since the functional $E$ is convex on the set of symmetrized functions and is strongly lower semi-continuous on $W_{0}^{1, p}(\Omega)$, it is weakly lower semi-continuous on the set of symmetrized functions, whence

$$
E(u) \leqslant \liminf _{n \rightarrow \infty} E\left(u_{n}\right)=\inf _{v \in \mathcal{M}} E(v) .
$$

Let $\cdot^{\star}$ denote the Steiner symmetrization with respect to $\{0\} \times \mathbb{R}^{N-k}$. By a theorem of Lions on compact embeddings of sets of symmetric functions [20] the sequence $\left(u_{n}^{\star}\right)$ is compact in $L^{q}(\Omega)$. Therefore, the sequence $\left(u_{n}\right)=\left(u_{n}^{\star *}\right)$ is compact in $L^{q}(\Omega)$.

Up to a subsequence, $u_{n} \rightarrow u$ almost everywhere. Since

$$
\lim _{s \rightarrow 0} \sup _{x \in \Omega} \frac{|f(x, s)|}{|s|^{p}}=0,
$$

for any $\varepsilon>0$, there exists $\gamma_{\varepsilon}>0$ such that

$$
|f(x, s)| \leqslant \varepsilon|s|^{p}+\gamma_{\varepsilon}|s|^{q} .
$$

By Fatou's Lemma and by the weak convergence of the sequence $\left(u_{n}\right)$ in $L^{p}(\Omega)$,

$$
\begin{aligned}
& \varepsilon\|u\|_{p}^{p}+\gamma_{\varepsilon}\|u\|_{q}^{q}+\int_{\Omega} f(x, u) \mathrm{d} x \leqslant \liminf _{n \rightarrow \infty} \int_{\Omega} \varepsilon\left|u_{n}\right|+\gamma_{\varepsilon}\left|v_{n}\right|^{q}-f\left(x, u_{n}\right) \mathrm{d} x \\
& \leqslant \varepsilon \liminf _{n \rightarrow \infty}\left\|u_{n}\right\|_{p}^{p}+\gamma_{\varepsilon}\|u\|_{q}^{q}+\liminf _{n \rightarrow \infty} \int_{\Omega} f\left(x, u_{n}\right) \mathrm{d} x .
\end{aligned}
$$

As $\varepsilon \rightarrow 0$ this becomes

$$
\int_{\Omega} f(x, u) \mathrm{d} x \leqslant \liminf _{n \rightarrow \infty} \int_{\Omega} f\left(x, u_{n}\right) \mathrm{d} x .
$$


The same argument holds also for $-f$, hence

$$
\int_{\Omega} f(x, u) \mathrm{d} x=\lim _{n \rightarrow \infty} \int_{\Omega} f\left(x, u_{n}\right) \mathrm{d} x=1,
$$

$u \in \mathcal{M}$ and $E(u)=\inf _{v \in \mathcal{M}} E(v)$.

\section{Acknowledgements}

The author is supported by a Research Fellow Grant of the "Fonds National de la Recherche Scientifique" (Belgium). He thanks Michel Willem for encouragements, discussions and careful reading.

\section{References}

[1] R.A. Adams, Sobolev Spaces, Pure Appl. Math., vol. 65, Academic Press, New York, 1975.

[2] A. Alvino, V. Ferone, G. Trombetti, P.-L. Lions, Convex symmetrization and applications, Ann. Inst. H. Poincaré Anal. Non Linéaire 14 (2) (1997) 275-293.

[3] A. Alvino, G. Trombetti, P.-L. Lions, On optimization problems with prescribed rearrangements, Nonlinear Anal. 13 (2) (1989) $185-220$.

[4] A. Baernstein II, A unified approach to symmetrization, in: A. Alvino, et al. (Eds.), Partial Equations of Elliptic Type, in: Sympos. Math., vol. 35, Cambridge University Press, Cambridge, 1995, pp. 47-49.

[5] H.J. Brascamp, E.H. Lieb, J.M. Luttinger, A general rearrangement inequality for multiple integrals, J. Funct. Anal. 17 (1974) $227-237$.

[6] F. Brock, A.Yu. Solynin, An approach to symmetrization via polarization, Trans. Amer. Math. Soc. 352 (4) (2000) $1759-1796$.

[7] A. Burchard, Cases of equality in the Riesz rearrangement inequality, Ann. of Math. (2) 143 (3) (1996) $499-527$.

[8] A. Burchard, Steiner symmetrization is continuous in $W^{1, p}$, Geom. Funct. Anal. 7 (5) (1997) 823-860.

[9] H. Busemann, The isoperimetric problem for Minkowski area, Amer. J. Math. 71 (1949) 743-762.

[10] J.A. Crowe, P.C. Rosenbloom, J.A. Zweibel, Rearrangements of functions, J. Funct. Anal. 66 (1986) $432-438$.

[11] B. Dacorogna, Direct Methods in the Calculus of Variations, Springer-Verlag, Berlin, 1989.

[12] B. Dacorogna, C.-E. Pfister, Wulff theorem and best constant in Sobolev inequality, J. Math. Pures Appl. (9) 71 (2) (1992) $97-118$.

[13] I. Ekeland, R. Temam, Convex Analysis and Variational Problems, Stud. Math. Appl., vol. 1, North-Holland Publishing Co., Amsterdam, 1976.

[14] H. Federer, Geometric Measure Theory, Springer-Verlag, New York, 1969.

[15] I. Fonseca, S. Müller, A uniqueness proof for the Wulff theorem, Proc. Roy. Soc. Edinburgh Sect. A 119 (1-2) (1991) $125-136$.

[16] B. Kawohl, On the shape of solutions to some variational problems, in: Nonlinear Analysis, Function Spaces and Applications, vol. 5, Prague, 1994, Prometheus, Prague, 1994, pp. 77-102.

[17] B. Kawohl, Rearrangements and Convexity of Level Sets in PDE, Lecture Notes in Math., vol. 1150, Springer-Verlag, Berlin, 1985.

[18] V.S. Klimov, On the symmetrization of anisotropic integral functionals, Izv. Vyssh. Uchebn. Zaved. Mat. (8) (1999) $26-32$.

[19] E.H. Lieb, M. Loss, Analysis, second ed., Grad. Stud. Math., vol. 14, American Mathematical Society, Providence, RI, 2001.

[20] P.-L. Lions, Symétrie et compacité dans les espaces de Sobolev, J. Funct. Anal. 49 (3) (1982) 315-334.

[21] J. Mossino, Inégalités isopérimétriques et applications en physique, Travaux en cours, Hermann, Paris, 1984.

[22] G. Pólya, G. Szegö, Isoperimetric Inequalities in Mathematical Physics, Princeton University Press, Princeton, NJ, 1951.

[23] J. Sarvas, Symmetrization of condensers in $n$-space, Ann. Acad. Sci. Fenn., Ser. A I 522 (1972) 1-44.

[24] K.R. Stromberg, Probability for Analysts, Chapman \& Hall Probability Series, Chapman \& Hall, New York, 1994. Lecture notes prepared by Kuppusamy Ravindran.

[25] G. Talenti, Best constant in Sobolev inequality, Ann. Mat. Pura Appl. (4) 110 (1976) 353-372.

[26] G. Talenti, On isoperimetric theorems of mathematical physics, in: P.M. Gruber, J. Wills (Eds.), Handbook of Convex Geometry B, Elsevier Science, Amsterdam, 1993, pp. 1131-1147.

[27] J.E. Taylor, Crystalline variational problems, Bull. Amer. Math. Soc. 84 (4) (1978) 568-588.

[28] J. Van Schaftingen, Universal approximation of symmetrizations by polarizations, Proc. Amer. Math. Soc. 134 (1) (2006) $177-186$.

[29] J. Van Schaftingen, M. Willem, Set transformations, symmetrizations and isoperimetric inequalities, in: V. Benci, A. Masiello (Eds.), Nonlinear Analysis and Applications to the Physical Sciences, Springer, 2004, pp. 135-152.

[30] M. Willem, Analyse fonctionnelle élémentaire, Cassini, Paris, 2003.

[31] W.P. Ziemer, Weakly Differentiable Functions: Sobolev Spaces and Functions of Bounded Variation, Grad. Texts in Math., vol. 120, SpringerVerlag, New York, 1989. 